MATHEMATICS OF COMPUTATION

Volume 81, Number 278, April 2012, Pages 687-713

S $0025-5718(2011) 02527-5$

Article electronically published on July 26, 2011

\title{
DISCONTINUOUS GALERKIN FINITE ELEMENT HETEROGENEOUS MULTISCALE METHOD FOR ELLIPTIC PROBLEMS WITH MULTIPLE SCALES
}

\author{
ASSYR ABDULLE
}

\begin{abstract}
An analysis of a multiscale symmetric interior penalty discontinuous Galerkin finite element method for the numerical discretization of elliptic problems with multiple scales is proposed. This new method, first described in [A. Abdulle, C.R. Acad. Sci. Paris, Ser. I 346 (2008)] is based on numerical homogenization. It allows to significantly reduce the computational cost of a fine scale discontinuous Galerkin method by probing the fine scale data on sampling domains within a macroscopic partition of the computational domain. Macroscopic numerical fluxes, an essential ingredient of discontinuous Galerkin finite elements, can be recovered from the computation on the sampling domains with negligible computation overhead. Fully discrete a priori error bounds are derived in the $L^{2}$ and $H^{1}$ norms.
\end{abstract}

\section{INTRODUCTION}

The modeling and the numerical simulation of physical processes in strongly heterogeneous media (e.g., deformation or diffusion in composite materials, flow in porous medium, etc.), pose major mathematical and computational challenges. In theory, the numerical approximation of such problems could be obtained by standard numerical methods as the finite element method (FEM), the finite difference method (FDM) or the finite volume method (FVM). However, the convergence of such methods requires a mesh size $h$ small enough to resolve the finest length scale of the problem (denoted here by $\varepsilon$ ), i.e., $h<\varepsilon$. Therefore, if $\varepsilon$ is small, microscopic computations often represent a prohibitive cost due to the high number of degrees of freedom. A cheap remedy is to neglect the fine scales of the problem in numerical computations. But macroscopic approaches which do not take into account the fine-scale physics are of limited interest, since the microscopic heterogeneities significantly affect the properties of the solution at larger scales. One thus has to rely on approaches combining microscopic and macroscopic descriptions of the problem.

Methods based on a coupling of macroscopic and microscopic solvers have become increasingly popular. We mention upscaling methods in structural mechanics 48, the quasicontinuum method [47, the gap-tooth scheme [40] and the heterogeneous multiscale method (HMM) 31. Multiscale FEMs for elliptic problems (based on multiscale basis functions) have been pioneered by Babŭska and Osborn [18, [19].

Received by the editor October 5, 2009 and, in revised form, January 29, 2011.

2010 Mathematics Subject Classification. Primary 65N30 65M60; Secondary 74Q05, 35J15.

Key words and phrases. Heterogeneous multiscale method, discontinuous Galerkin methods, a priori error analysis, fully discrete error, elliptic homogenization.

(C)2011 American Mathematical Society Reverts to public domain 28 years from publication 
Recent developments, mainly for elliptic problems with multiple scales, include [15, [35], 37, [42], 48] (see also the references in these papers).

The framework of the HMM is used to construct the new method proposed in this paper. The HMM is based on the assumption that a macroscopic description of the multiscale problem exists but may not be explicitly given. This macroscopic problem is, however, solved directly by a macro FEM on a coarse mesh. The (unknown) effective data of the macroscopic problems are extracted on the fly by solving microscopic problems (micro FEM) on sampling domains within the coarse mesh. For homogenization problems, the HMM is related to so-called numerical homogenization methods, relying on the solution of local problems to derive an effective equation, where the small scales have been averaged out. However, the simultaneous coupling of macroscopic and microscopic solvers offers advantages for the analysis (quantify the propagation of error across scales), for coupling various type of FEM at various scales, for switching locally to the fine scale solver (e.g., near cracks or fractures) and for designing numerical software [12, 6], 7].

The analysis of the finite element heterogeneous multiscale method (FE-HMM) has been given in [32] and [9] assuming exact solutions of the micro solver. The first fully discrete a priori analysis for elliptic and elastic problems has been proposed in [3] and 5, respectively. The framework of the HMM is particularly flexible to allow different types of methods to be used at different scales. In 10, a coupling of a FEM for the macroscopic solver with a pseudo-spectral method for the micro solver has been proposed allowing near optimal computational complexity for problems involving oscillatory coefficients with sufficient regularity. Finally, crucial for practical computations is the ability to assess the quality of a computed solution and to refine the grid in elements that contribute most to the global error. This has been studied in [13, 7].

Local conservation properties in the numerical approximation and flexibility in meshing (e.g., hanging nodes, local refinements) are desirable for many problems. A popular methodology to achieve these aims is to use (local) discontinuous approximations in the FE space as, for example, in the discontinuous Galerkin (DG) FEMs. Such methods have been extensively studied for hyperbolic problems, advectiondiffusion and diffusion problems [16, 17, 26, 27, [38, 49. While a large body of literature is available for DG methods applied to single scale problems, the construction and analysis of multiscale DG methods have rarely been addressed. We note that a DG method in the framework of the HMM for hyperbolic and parabolic one-dimensional scalar problems has been proposed in [25. The formulation of this latter method (based on the local discontinuous Galerkin method) differs from the method proposed in the present paper. Another DG-FEM for multiscale elliptic problems has been proposed in [1. This method is constructed in the framework of the multiscale finite element (MsFEM) framework of [35. This method, which has not yet been analyzed, also differs significantly from the method proposed here. In this paper we construct and analyze a numerical method, the discontinuous Galerkin heterogeneous multiscale method (DG-HMM), combining HMM with the symmetric interior penalty (IP) discontinuous Galerkin FEM (DG-FEM). To the best of our knowledge, this is the first analysis of a multiscale DG method for elliptic homogenization problems. The attractive properties of the DG-FEM as local mass and flux conservation or flexibility in meshing, will be inherited by our multiscale method at the macroscopic level. The DG-HMM, has first been announced in [11. 
In this paper we give the full proof, improve and extend the results stated in [11] to a more general framework. Non-optimal micro a priori error bounds were stated in [11. Here, optimal fully discrete error bounds in the $L^{2}$ and $H^{1}$ norms are derived.

Although the implementation of our method and the proof of the existence of a numerical solution do not require any assumption on the type of heterogeneities, the analysis of the convergence rates relies on local periodicity of the conductivity tensor (piecewise constant in the slow variable). We note that periodic coefficients have been used for the analysis of multiscale methods by many authors [15, 35, [36], 42], 45].

Our method is not limited to elliptic problems and can readily be applied to parabolic problems. It is known that for such problems, the mass matrix is blockdiagonal when using a DG-FEM (even diagonal if choosing orthogonal basis functions). Thus, a multiscale discretization in space can be easily combined with explicit stabilized solvers in time such as the ROCK methods [8], 2] to obtain a fully discrete explicit coarse-grained algorithm for time dependent multiscale parabolic problems. Besides the symmetric interior penalty DG-FEM, other types of DG methods could be used in the framework developed below. The extension of the results of [3] for non-symmetric elliptic problems obtained in 29] could, for example, be used to construct DG methods for non-symmetric problems.

The paper is organized as follows. In subsection 2.1 we discuss the multiscale model problem and briefly recall the HMM developed so far for this type of problems. In subsection 2.2 we briefly recall the FE-HMM and introduce in section 3 our new discontinuous Galerkin method for elliptic multiscale problems. The well-posedness of our method is proved in section 4 and a priori error estimates for locally periodic oscillating tensor and various coupling conditions between macro and micro methods are given in section [5. A generalization to higher order is discussed. Concluding remarks are given at the end of the paper in section 6 .

Notations. In what follows, $C, \tilde{C}$ or $\bar{C}$ denote generic positive constants, independent of $\varepsilon$, whose values can change at any occurrence but depend only on the quantities which are indicated explicitly. We will consider the usual Sobolev space $W^{s, p}(\Omega)$. For $p=2$ we use the notation $H^{s}(\Omega)$ and $H_{0}^{1}(\Omega)$, and denote by $W_{p e r}^{1}(Y)=\left\{v \in H_{p e r}^{1}(Y) ; \int_{Y} v d x=0\right\}$, where $H_{p e r}^{s}(Y)$ is defined as the closure of $\mathcal{C}_{\text {per }}^{\infty}(Y)$ (the subset of $\mathcal{C}^{\infty}\left(\mathbb{R}^{d}\right)$ of periodic functions in the unit cube $\left.Y=(0,1)^{d}\right)$ for the $H^{s}$ norm. For a matrix $A \in \mathbb{R}^{d \times d}$ with entries $a_{i j}$, we denote its Frobenius norm by $\|A\|_{F}:=\sqrt{\operatorname{trace}\left(A^{T} A\right)}=\sqrt{\sum_{i j} a_{i j}^{2}}$.

\section{Model PRoblem AND Finite ElEMENT HeterogeneOUs MULTISCALE METHOD}

In this section we briefly describe the homogenization problems that we consider in this paper and we recall the (continuous) finite element heterogeneous multiscale method (FE-HMM).

2.1. Homogenization problem. We consider the second-order elliptic problem in a convex polygonal domain $\Omega \subset \mathbb{R}^{d}, d \leq 3$,

$$
-\nabla \cdot\left(a^{\varepsilon} \nabla u^{\varepsilon}\right)=f \text { in } \Omega, \quad u^{\varepsilon}=0 \text { on } \partial \Omega,
$$


where we assume that the family of tensors, indexed by $\varepsilon$, are symmetric, satisfy $a^{\varepsilon}(x) \in\left(L^{\infty}(\Omega)\right)^{d \times d}$, and are uniformly elliptic and bounded, i.e.,

$$
\exists \lambda, \Lambda>0 \text { such that } \lambda|\xi|^{2} \leq a^{\varepsilon}(x) \xi \cdot \xi \leq \Lambda|\xi|^{2}, \forall \xi \in \mathbb{R}^{d} \text {, a.e. } x \in \Omega \text { and } \forall \varepsilon>0,
$$

where $\varepsilon$ represents a small scale in the problem that characterizes the multiscale nature of the tensor $a^{\varepsilon}(x)$. We further assume that $f \in L^{2}(\Omega)$. An application of the Lax-Milgram theorem gives us a family of solutions $\left\{u^{\varepsilon}\right\}$ which are bounded in $H_{0}^{1}(\Omega)$. Without making any further assumption on the heterogeneities of the tensor $a^{\varepsilon}(x)$ using the notions of $G$ - convergence introduced by De Giorgi and Spagnolo $[28]^{1}$ it is possible to show that there exists a symmetric tensor $a^{0}(x)$ and a subsequence of $\left\{u^{\varepsilon}\right\}$ which weakly converges to an element $u^{0} \in H_{0}^{1}(\Omega)$, where $u^{0}$ is the solution of the so-called homogenized or upscaled problem

$$
-\nabla \cdot\left(a^{0} \nabla u^{0}\right)=f \text { in } \Omega, \quad u^{0}=0 \text { on } \partial \Omega .
$$

It can also be shown that $a^{0}(x)$ (called the homogenized tensor) again satisfies $\lambda|\xi|^{2} \leq a^{0}(x) \xi \cdot \xi \leq \Lambda|\xi|^{2}, \forall \xi \in \mathbb{R}^{d}$ and problem (2.3) thus has a unique solution. If $a^{\varepsilon}(x)$ has more spatial structure, e.g., if $a^{\varepsilon}(x)=a(x, x / \varepsilon)$ and is periodic in its second argument, then classical results in homogenization theory (see [39, 24]) show that the whole sequence $\left\{u^{\varepsilon}\right\}$ weakly converges to an element $u^{0} \in H_{0}^{1}(\Omega)$ and the homogenized tensor $a^{0}(x)$ at $x \in \Omega$ can be characterized by a suitable average of the solutions of $d$ boundary value problems, the "cell problems". In the latter case, one has in general infinitely many cell problems to solve in order to compute the matrix-valued function $a^{0}(x)$. Notice that in the above problem we have chosen zero Dirichlet boundary conditions for simplicity. The numerical method constructed in this paper can accommodate non-zero Dirichlet, Neumann, or mixed boundary conditions without difficulties (with obvious changes in the bilinear form). Our analysis also applies to these other types of boundary conditions.

2.2. The FE-HMM. A direct approach for numerical homogenization of (2.1) which takes the fine scale tensor $a^{\varepsilon}(x)$ as input coefficient has been recently proposed in the framework of the HMM [31]. In a finite element context, the FE-HMM was analyzed in [9], 3], [10], 32]. The method aims at capturing the homogenized (coarse) solution of (2.1) without computing $a^{0}(x)$ explicitly. The method relies on macro FEM based on quadrature points within a macro triangulation and micro FEM defined on sampling domains centered at the quadrature points.

Macro finite element space. We consider

$$
S_{H}^{\ell}\left(\Omega, \mathcal{T}_{H}\right)=\left\{v^{H} \in H_{0}^{1}(\Omega) ;\left.u^{H}\right|_{K} \in \mathcal{R}^{\ell}(K), \forall K \in \mathcal{T}_{H}\right\},
$$

with macro elements $K \in \mathcal{T}_{H}$. We denote $H=\max _{K \in \mathcal{T}_{H}} H_{K}$, where $H_{K}$ is the diameter of the element $K$ and $\mathcal{T}_{H}$ is the macromesh of the domain $\Omega$. Here $H$ is allowed to be much larger than $\varepsilon$. The polynomial space $\mathcal{R}^{\ell}$ denotes either $\mathcal{P}^{\ell}$ the space of piecewise polynomials on the element $K$ of total degree $\ell$ if $K$ is a simplicial element (triangle if $d=2$, tetrahedron if $d=3$ ), or $\mathcal{Q}^{\ell}$ the space of piecewise polynomials on the element $K$ of degree $\ell$ in each variable, if $K$ is a quadrilateral element (quadrilateral if $d=2$, hexahedron if $d=3$ ).

\footnotetext{
${ }^{1}$ A generalization of this notion, called $H$-convergence has been introduced by Murat and Tartar 43 .
} 
Quadrature formulas. We define in each macro element $K \in \mathcal{T}_{H} \quad J$ macro quadrature points $x_{K_{j}}$ and a quadrature formula (QF) $\left\{x_{K_{j}}, \omega_{K_{j}}\right\}_{j=1}^{J}$, where $\omega_{K_{j}}$ are the quadrature weights. Centered around each macro quadrature point we define a sampling domain $K_{\delta_{j}}=x_{K_{j}}+\delta I$, where $I=(-1 / 2,1 / 2)^{d}$ and $\delta \geq \varepsilon$. These sampling domains are the physical domains for the micro FEMs. The QF on $K$ needs to be appropriately chosen to have optimal convergence results. More precisely, for a reference element $\hat{K}$ we consider a reference quadrature formula $\left\{\hat{x}_{j}, \hat{\omega}_{j}\right\}_{j=1}^{J}$ and require

(Q1) $\hat{\omega}_{j}>0, j=1, \ldots, J, \quad \sum_{j=1}^{J} \hat{\omega}_{j}\left|\nabla \hat{q}\left(\hat{x}_{j}\right)\right|^{2} \geq \hat{\lambda}\|\nabla \hat{q}\|_{L^{2}(\hat{K})}^{2}, \forall \hat{q}(x) \in \mathcal{R}^{\ell}(\hat{K}) ;$

(Q2) $\int_{\hat{K}} \hat{q}(x) d x=\sum_{j=1}^{J} \hat{\omega}_{j} \hat{q}\left(\hat{x}_{j}\right), \forall \hat{q}(\hat{x}) \in \mathcal{R}^{\sigma}(\hat{K})$, where $\sigma=\max (2 \ell-2, \ell)$ if $\hat{K}$ is a simplicial FE, or $\sigma=\max (2 \ell-1, \ell+1)$ if $\hat{K}$ is a rectangular $\mathrm{FE}$.

The quadrature weights and integration points on $K \in \mathcal{T}_{H}$ are then given by $x_{K_{j}}=F_{K}\left(\hat{x}_{j}\right), \omega_{K_{j}}=\hat{\omega}_{j} \operatorname{det}\left(\partial F_{K}\right), j=1, \ldots, J$, where $F_{K}$ is a $C^{1}$-diffeomorphism such that $K=F_{K}(\hat{K})$.

Macro bilinear form. For $v^{H}, w^{H} \in S_{H}^{\ell}\left(\Omega, \mathcal{T}_{H}\right)$ we define

$$
B\left(v^{H}, w^{H}\right)=\sum_{K \in \mathcal{T}_{H}} \sum_{j=1}^{J} \frac{\omega_{K_{j}}}{\left|K_{\delta_{j}}\right|} \int_{K_{\delta_{j}}} a^{\varepsilon}(x) \nabla v_{K_{j}}^{h} \cdot \nabla w_{K_{j}}^{h} d x,
$$

where $v_{K_{j}}^{h}, w_{K_{j}}^{h}$ are appropriate micro-functions defined on sampling domains $K_{\delta_{j}}$ (see below) and the factor $\left|K_{\delta_{j}}\right|$ gives the appropriate weight for the contribution of the integrals defined on $K_{\delta_{j}}$.

Micro solver. For a macro element $K$, consider $K_{\delta_{j}}, j=1, \ldots, J$, the sampling domains included in $K$. For each sampling domain $K_{\delta_{j}}$ we consider a micromesh $\mathcal{T}_{h}$, a partition of $K_{\delta_{j}}$ in simplicial or quadrilateral FEs. We then determine the additive contribution to the macro stiffness matrix by computing micro-functions $v_{K_{j}}^{h}$ (and $w_{K_{j}}^{h}$ ) obtained by solving micro-problems on sampling domains $K_{\delta_{j}}, j=1, \ldots, J$. The micro-problems read as follows: find $v_{K_{j}}^{h}$ such that $\left(v_{K_{j}}^{h}-v_{\text {lin } K_{j}}^{H}\right) \in S^{q}\left(K_{\delta_{j}}, \mathcal{T}_{h}\right)$ and

$$
\int_{K_{\delta_{j}}} a^{\varepsilon}(x) \nabla v_{K_{j}}^{h} \cdot \nabla z^{h} d x=0 \quad \forall z^{h} \in S^{q}\left(K_{\delta_{j}}, \mathcal{T}_{h}\right)
$$

where

$$
v_{l i n, K_{j}}^{H}(x)=v^{H}\left(x_{K_{j}}\right)+\left(x-x_{K_{j}}\right) \cdot \nabla v^{H}\left(x_{K_{j}}\right)
$$

is a linearization of the macro function $v^{H}$ at the integration point $x_{K_{j}}$ (see [6], 32 for details) and

$$
S^{q}\left(K_{\delta_{j}}, \mathcal{T}_{h}\right)=\left\{z^{h} \in W\left(K_{\delta_{j}}\right) ;\left.z^{h}\right|_{T} \in \mathcal{R}^{q}(T), T \in \mathcal{T}_{h}\right\},
$$

where the choice of $W\left(K_{\delta_{j}}\right)$ determines the coupling condition or boundary conditions used for computing the micro-functions $v_{K_{j}}^{h}\left(\right.$ or $w_{K_{j}}^{h}$ ). Several choices are possible for the coupling condition. We consider

$$
W\left(K_{\delta_{j}}\right)=W_{p e r}^{1}\left(K_{\delta_{j}}\right)=\left\{v \in H_{p e r}^{1}\left(K_{\delta_{j}}\right) ; \int_{K_{\delta_{j}}} v d x=0\right\},
$$

for a periodic coupling or

$$
W\left(K_{\delta_{j}}\right)=H_{0}^{1}\left(K_{\delta_{j}}\right)
$$


for a coupling through Dirichlet boundary conditions. We will sometimes refer to these couplings as "periodic coupling" or "Dirichlet coupling".

Variational problem. The macro numerical solution for the problem (2.3) is defined by the following variational problem: find $u^{H} \in S_{H}^{\ell}\left(\Omega, \mathcal{T}_{H}\right)$ such that

$$
B\left(u^{H}, v^{H}\right)=\int_{\Omega} f v^{H} d x \quad \forall v^{H} \in S_{H}^{\ell}\left(\Omega, \mathcal{T}_{H}\right) .
$$

The primary goal of this method is to capture the effective solution $u^{0}$ of (2.3). As the method not only depends on the macromesh (of size $H$ ) but also on the micromesh (of size $h$ ) $H, h \rightarrow 0$ is needed for convergence. The rate at which both meshes should be refined has been analyzed in [3. This is crucial to have the optimal accuracy with the smallest computational cost. As local information on the small scale solution is available in the sampling domains $K_{\delta_{j}} \subset K$ (through the micro-functions), a proper reconstruction in a post-processing step allows to define an approximation of the fine scale solution $u^{\varepsilon}$ of the equation (2.1). The accuracy of such a reconstruction has also been analyzed in [3].

\section{Discontinuous Galerkin heterogeneous multiscale method}

In this section we introduce our multiscale method combining the heterogeneous multiscale method and the interior penalty discontinuous Galerkin FEM. For simplicity of the presentation we will consider simplicial piecewise linear micro and macro finite elements. We notice that higher order FEs for the FE-HMM have been considered and analyzed in [32, [10, 6]. Higher order FEs for the micro method on the sampling domains can readily be used for the discontinuous Galerkin method presented below and the analysis of [10, 6] can be carried over to the present situation with essentially no changes. However, a generalization of the method given below is needed to accommodate higher order simplicial macro FEs or quadrilateral FEs. This will be discussed in Section 5.4 .

When considering linear macro FEs, $J=1$ and hence we denote $x_{1, K}$ (located at the barycenter) by $x_{K}$ and $\omega_{1, K}=|K|$ by $\omega_{K}$. Likewise, the sampling domains are denoted by $K_{\delta}$ and the micro-functions by $v_{K}^{h}$.

3.1. The interior penalty DG-FEM. For the convenience of the reader we shortly recall the standard (single-scale) DG-FEM.

In this subsection, there are no macromeshes and micromeshes, and $\mathcal{T}_{h}$ denotes a shape-regular partition of $\Omega$. The elements of the partition are denoted by $T$ here, i.e., $\bar{\Omega}=\bigcup_{T \in \mathcal{T}_{h}} T$ and $h=\max _{T \in \mathcal{T}_{h}} h_{T}$, where $h_{T}$ is the size of $T 2$ We denote by $\mathcal{E}$ the set of all open $(d-1)$-dimensional interfaces (faces or edges) of the elements of $\mathcal{T}_{h}$. Since hanging nodes are permitted, elements of $\mathcal{E}$ will be defined as the smallest common interfaces of neighboring elements $T \in \mathcal{T}_{h}$. We further denote by $\mathcal{E}_{i n t}$ the set of all interior interfaces and by $\mathcal{E}_{B}$ the set of all boundary interfaces. Thus, $\mathcal{E}=\mathcal{E}_{\text {int }} \cup \mathcal{E}_{B}$.

Let $e \in \mathcal{E}_{\text {int }}$ be an interface shared by two neighboring elements $T^{+}$and $T^{-}$. For a piecewise smooth function $v$, we denote by $v_{+}, v_{-}$its trace from within $T^{+}, T^{-}$,

\footnotetext{
${ }^{2}$ In the previous subsection and in the remaining part of the paper $\mathcal{T}_{h}$ denotes the micro triangulation of the sampling domains $K_{\delta_{j}}$. This should not be confused with the mesh used in this subsection to denote the partition of $\Omega$.
} 
respectively, and define the average and the jump of $v$ by

$$
\{v\}=\frac{1}{2}\left(v_{+}+v_{-}\right), \quad \llbracket v \rrbracket=v_{+} n_{T^{+}}+v_{-} n_{T^{-}},
$$

respectively, where $n_{T^{ \pm}}$denotes the unit outward normal vectors on the interface $T^{ \pm}$. Similarly, for a piecewise smooth vector-valued function $q$, we define the average and the jump by

$$
\{q\}=\frac{1}{2}\left(q_{1}+q_{2}\right), \quad \llbracket q \rrbracket=q_{1} \cdot n_{+}+q_{2} \cdot n_{-},
$$

respectively. For an interface on the boundary $\partial \Omega$ we define

$$
\{v\}=v, \llbracket v \rrbracket=v n \text { and }\{q\}=q, \llbracket q \rrbracket=q \cdot n,
$$

where $n$ is the unit outward normal vector on $\partial \Omega$. Notice that the jump of a scalar function is a vector-valued function, while the jump of a vector-valued function is a scalar one.

Relaxing the interelement continuity requirement for a standard FE space, we consider

$$
V_{h}\left(\Omega, \mathcal{T}_{h}\right)=\left\{u^{h} \in L^{2}(\Omega) ;\left.u^{h}\right|_{T} \in \mathcal{P}^{1}(T), \forall T \in \mathcal{T}_{h}\right\},
$$

where $\mathcal{P}^{1}(T)$ is the space of linear polynomials on the triangle $T$. We also define the piecewise Sobolev space

$$
H^{k}\left(\mathcal{T}_{h}\right):=\prod_{T \in \mathcal{T}_{h}} H^{k}(T)=\left\{v \in L^{2}(\Omega) ;\left.v\right|_{T} \in H^{k}(T) \forall T \in \mathcal{T}_{h}\right\}
$$

We note that $V_{h}\left(\Omega, \mathcal{T}_{h}\right) \subset H^{1}\left(\mathcal{T}_{h}\right)$. The classical interior penalty DG-FEM can be obtained as follows: we consider an arbitrary element $T$ of our triangulation $\mathcal{T}_{h}$; multiply the problem (2.1) by a smooth test function $v$ and integrate by parts (using $a^{\varepsilon} \nabla u^{\varepsilon} \in H(\operatorname{div}, \Omega)$ to obtain

$$
-\int_{T} \nabla \cdot\left(a^{\varepsilon} \nabla u^{\varepsilon}\right) v d x=\int_{T} a^{\varepsilon} \nabla u^{\varepsilon} \cdot \nabla v d x-\int_{\partial T} a^{\varepsilon} \nabla u^{\varepsilon} \cdot n_{T} v d s,
$$

where $n_{T}$ is the outward normal. Summing over $T \in \mathcal{T}_{h}$ yields

$$
\int_{\Omega} a^{\varepsilon} \nabla u^{\varepsilon} \cdot \nabla v d x-\sum_{T \in \mathcal{T}_{h}} \int_{\partial T} a^{\varepsilon} \nabla u^{\varepsilon} \cdot n_{T} v d s=\int_{\Omega} f v d x
$$

and using the above notation we can rewrite (3.5) as

$$
\int_{\Omega} a^{\varepsilon} \nabla u^{\varepsilon} \cdot \nabla v d x-\sum_{e \in \mathcal{E}} \int_{e}\left\{a^{\varepsilon} \nabla u^{\varepsilon}\right\} \llbracket v \rrbracket d s=\int_{\Omega} f v d x,
$$

where we notice that $a^{\varepsilon} \nabla u^{\varepsilon} \in H\left(\operatorname{div}, T^{+} \cup T^{-}\right)$implies $a^{\varepsilon} \nabla u^{\varepsilon} \in H\left(\operatorname{div}, T^{ \pm}\right)$and $\left(a^{\varepsilon} \nabla u^{\varepsilon}\right){ }_{1} n_{T^{+}}+\left(a^{\varepsilon} \nabla u^{\varepsilon}\right){ }_{2} n_{T^{-}}=0$. This in turn implies that

$$
\left(a^{\varepsilon} \nabla u^{\varepsilon}\right)_{1} n_{T^{+}} v_{+}+\left(a^{\varepsilon} \nabla u^{\varepsilon}\right)_{2} n_{T^{-}} v_{-}=\left\{a^{\varepsilon} \nabla u^{\varepsilon}\right\} \llbracket v \rrbracket .
$$

Since the exact solution of (2.1) is in $H_{0}^{1}(\Omega)$ we have $\llbracket u^{\varepsilon} \rrbracket=0$ and we can make the bilinear form (3.6) symmetric by adding $-\sum_{e \in \mathcal{E}} \int_{e}\left\{a^{\varepsilon} \nabla v\right\} \llbracket u^{\varepsilon} \rrbracket d s$ (assuming the existence of a trace for $a^{\varepsilon} \nabla v$ ). Finally, to have a stable method one adds a penalty 
term. The finite-dimensional version leads to the interior penalty DG-FEM (see [17) which reads as follows: find $u^{h} \in V_{h}\left(\Omega, \mathcal{T}_{h}\right)$ such that

$$
\begin{gathered}
\int_{\Omega} a^{\varepsilon} \nabla u^{h} \cdot \nabla v^{h} d x-\sum_{e \in \mathcal{E}} \int_{e}\left(\left\{a^{\varepsilon} \nabla u^{h}\right\} \llbracket v^{h} \rrbracket+\left\{a^{\varepsilon} \nabla v^{h}\right\} \llbracket u^{h} \rrbracket\right) d s \\
+\sum_{e \in \mathcal{E}} \int_{e} \mu \llbracket u^{h} \rrbracket \llbracket v^{h} \rrbracket d s=\int_{\Omega} f v^{h} d x \quad \forall v^{h} \in V_{h}\left(\Omega, \mathcal{T}_{h}\right),
\end{gathered}
$$

where $\mu=\alpha h_{e}^{-1}$ with $\alpha>0$ independent of the meshsizes and $h_{e}$ is the interface size with the aforementioned convention for hanging nodes. The last term in the left-hand side of (3.7) is referred to as the interior penalty (IP) or stabilization term, where $\mu$ (the penalty weighting function) penalizes the jumps of $u^{h}$ and $v^{h}$ over the interfaces of $\mathcal{T}_{h}$.

Here and in what follows the gradient $\nabla$ should be understood as a broken gradient when dealing with functions discontinuous over $T \in \mathcal{T}_{h}$. The choice of $\alpha$ is dictated by stability requirements. The analysis of this method as well as the analysis of many other methods based on discontinuous Galerkin FE spaces for the Laplace problem are discussed in [17].

Remark 3.1. For multiscale problems such as (2.1), in addition to the requirement $h<\varepsilon$ (needed for convergence which can be prohibitive in terms of computational costs if $\varepsilon$ is small), we also need regularity on $a^{\varepsilon}$ to be able to define the numerical flux $a^{\varepsilon} \nabla v^{h}$ on $\partial T$ (existence of a trace). Such regularity on $a^{\varepsilon}$ may not be realistic for many problems with rough coefficients. In the method described below, the numerical multiscale flux will only involve a piecewise constant tensor based on a suitable average of $a^{\varepsilon}$ on sampling domains. Thus, we avoid the issue of the existence of traces of $a^{\varepsilon}$ on $\partial T$.

3.2. The heterogeneous multiscale method based on DG-FEM. In this section we derive a DG-HMM based on the macro DG space

$$
V_{H}\left(\Omega, \mathcal{T}_{H}\right)=\left\{u^{H} \in L^{2}(\Omega) ;\left.u^{H}\right|_{K} \in \mathcal{P}^{1}(K), \forall K \in \mathcal{T}_{H}\right\},
$$

where $\mathcal{P}^{1}(K)$ is the space of linear polynomials on the triangle $K$.

Since $H$ is allowed to be much larger than $\varepsilon$ we need to modify the bilinear form (3.7). The first term in (3.7) can be treated as in (2.5). In what follows we concentrate on the modeling of the macroscopic jump terms. We show that this can be done by using only the information from the micro-problems (2.6) needed anyway for assembling the stiffness matrix of the FE-HMM. For this purpose, we define for each interior interface $e$ of two elements $K^{+}, K^{-}$with sampling domains $K_{\delta}^{+}, K_{\delta}^{-}$and for an integrable function or vector-valued function $\xi\left(\xi_{ \pm}=\left.\xi\right|_{K^{ \pm}}\right)$an average of multiscale fluxes as

$$
\{\bar{\xi}\}=\frac{1}{2}\left(\frac{1}{\left|K_{\delta}^{+}\right|} \int_{K_{\delta}^{+}} \xi_{+} d x+\frac{1}{\left|K_{\delta}^{-}\right|} \int_{K_{\delta}^{-}} \xi_{-} d x\right) .
$$

For a boundary interface $e \in \mathcal{E}_{B}$ of a triangle $K$ with sampling domain $K_{\delta}$ we set

$$
\{\bar{\xi}\}=\left(\frac{1}{\left|K_{\delta}\right|} \int_{K_{\delta}} \xi d x\right) .
$$


We then define the following macro bilinear form on $V_{H}\left(\Omega, \mathcal{T}_{H}\right) \times V_{H}\left(\Omega, \mathcal{T}_{H}\right)$

$$
\begin{aligned}
B_{D G}\left(v^{H}, w^{H}\right) & =\sum_{K \in \mathcal{T}_{H}} \frac{|K|}{\left|K_{\delta}\right|} \int_{K_{\delta}} a^{\varepsilon} \nabla v_{K}^{h} \cdot \nabla w_{K}^{h} d x-\int_{\Gamma}\left(\left\{\overline{a^{\varepsilon} \nabla v^{h}}\right\} \llbracket w^{H} \rrbracket\right. \\
& \left.+\left\{\overline{a^{\varepsilon} \nabla w^{h}}\right\} \llbracket v^{H} \rrbracket\right) d s+\int_{\Gamma} \mu \llbracket v^{H} \rrbracket \llbracket w^{H} \rrbracket d s
\end{aligned}
$$

where we used the notation

$$
\int_{\Gamma} \cdot=\sum_{e \in \mathcal{E}} \int_{e}
$$

and where $v_{K}^{h}, w_{K}^{h}$ are given by the solution of the micro problems (2.6) ( $q=1$ for piecewise linear micro FEs). Notice that for the averages $\left\{\overline{a^{\varepsilon} \nabla v^{h}}\right\}$, we skipped the superscript notation $K$ as these quantities are defined on the interface of two elements. As in section 3.1 the penalty weighting function $\mu$ is defined by $\left.\mu\right|_{e}=$ $\mu_{e}=\alpha H_{e}^{-1}$, where $\alpha$ is a positive parameter independent of the local mesh size. We recall that $J=1$ and $x_{K_{j}}=x_{K}$ is located at the barycenter of $K$ and $\omega_{1, K}=|K|$. In case of hanging nodes, similarly as discussed before, $\left.H\right|_{e}=\min \left\{H_{K^{+}}, H_{K^{-}}\right\}$for an interface $e$ between two macro elements $K^{+}, K^{-}$.

Remark 3.2. In the above formulation the micro FE space (2.8) can be of arbitrary order. When using piecewise linear macro FEs, linear micro FEs are reasonable as the error analysis shows that the contribution of the micro-discretization contributes quadratically to the global error. For higher order macro FEs (see Section 5.4) higher order micro FEs can be used provided that the regularity of the small scales allows to take advantage of high order micro methods.

The macro solution of the DG-HMM is then defined by the following variational problem: find $u^{H} \in V_{H}\left(\Omega, \mathcal{T}_{H}\right)$ such that

$$
B_{D G}\left(u^{H}, v^{H}\right)=\int_{\Omega} f v^{H} d x, \quad \forall v^{H} \in V_{H}\left(\Omega, \mathcal{T}_{H}\right) .
$$

For the implementation of the above method, the usual elementwise assembly for FEM can be used. Expanding the unknown function $u^{H}$ in the basis of the macro FE space, the solutions of the micro problems (2.6) are then constrained by the local basis functions (we refer to [12] for details on the implementation).

Let us make some comments on this multiscale method. First, instead of solving the fine scale problem on the whole computational domain (as required for (3.7) since $h<\varepsilon$ ) we only solve it on sampling domains $K_{\delta}$ in the DG-HMM, hence we have a significant computational saving. Second, it is not required to be able to extend $a^{\varepsilon}$ on $\partial K$ (as was needed in (3.7)) as our multiscale fluxes are based on averaged quantities. Third, the macroscale fluxes are computed with minimal overhead using micro-functions already available from the computation of the first term in the DG-HMM bilinear form (3.10).

\section{Existence and uniqueness of a solution of the DG-HMM}

In this section we first show that (3.11) has a unique solution. This can be done with minimal assumptions on the oscillating tensor $a^{\varepsilon}$ (of course we need uniform boundedness and ellipticity).

First, we need to define the appropriate space for the analysis. We consider

$$
V(H)=V_{H}\left(\Omega, \mathcal{T}_{H}\right)+H_{0}^{1}(\Omega) \cap H^{2}(\Omega) \subset H^{2}\left(\mathcal{T}_{H}\right),
$$


with a mesh-dependent norm [17,

$$
\|v\|:=\left(\|\nabla v\|_{L^{2}(\Omega)}^{2}+\sum_{K \in \mathcal{T}_{H}} H_{K}^{2}|v|_{2, K}^{2}+|v|_{*}^{2}\right)^{1 / 2},
$$

where $H_{K}$ denotes the size of the element $H$ and where we used the notations

$$
\begin{gathered}
|v|_{m, K}^{2}=\sum_{|\alpha|=m}\left\|\partial^{\alpha} v\right\|_{L^{2}(K)}, \\
\|\nabla v\|_{L^{2}(\Omega)}^{2}=\sum_{K \in \mathcal{T}_{H}}|v|_{1, K}^{2}, \\
|v|_{*}^{2}=\sum_{e \in \mathcal{E}}\left\|\mu_{e}^{1 / 2} \llbracket v \rrbracket\right\|_{L^{2}(e)}^{2} .
\end{gathered}
$$

The function $\mu_{e}=\alpha H_{e}^{-1}$ is as previously defined. From the discrete Poincaré inequality [16, Lemma 2.1]

$$
\|v\|_{L^{2}(\Omega)} \leq C\left(\|\nabla v\|_{L^{2}(\Omega)}^{2}+|v|_{*}^{2}\right)
$$

we see that (4.2) is a norm on $V(H)$. Clearly, on $V\left(\Omega, \mathcal{T}_{H}\right)$ the norm (4.2) reduces to $\left(\|\nabla v\|_{L^{2}(\Omega)}^{2}+|v|_{*}^{2}\right)^{1 / 2}$. This is also a norm on $V(H)$. The additional term in (4.2) is needed to have stability on $V(H)$ [17.

We show now the existence and uniqueness of the solution of (3.11), where the micro solutions of (2.6) are constrained either by periodic or Dirichlet boundary conditions (see (2.8)). The first lemma is an energy equivalence for the constrained micro solutions.

Lemma 4.1. Let $v^{H} \in V_{H}\left(\Omega, \mathcal{T}_{H}\right)$ and let $v_{K}^{h}$ be the solution of (2.6) with boundary conditions given by (2.9) or (2.10). Assume that (2.2) holds. Then,

$$
\left\|\nabla v^{H}\right\|_{L^{2}\left(K_{\delta}\right)} \leq\left\|\nabla v_{K}^{h}\right\|_{L^{2}\left(K_{\delta}\right)} \leq \frac{\Lambda}{\lambda}\left\|\nabla v^{H}\right\|_{L^{2}\left(K_{\delta}\right)},
$$

where $\lambda, \Lambda$ are defined in (2.2).

Proof. See [3, Proposition 3.2].

Remark 4.2. The following generalization will be useful for the generalization of the DG-HMM to higher order macro FEs. Consider $v^{H} \in V_{H}^{\ell}\left(\Omega, \mathcal{T}_{H}\right)=\left\{v^{H} \in\right.$ $\left.L^{2}(\Omega) ;\left.v^{H}\right|_{K} \in P^{\ell}(K), \forall K \in \mathcal{T}_{H}\right\}$, and $v_{K_{j}}^{h}$ the solution of (2.6) in a sampling domain $K_{\delta_{j}} \subset K$ constrained by $v_{\text {lin, } K_{j}}^{H}$ (see (2.7)), then

$$
\left\|\nabla v_{l i n, K_{j}}^{H}\right\|_{L^{2}\left(K_{\delta_{j}}\right)} \leq\left\|\nabla v_{K_{j}}^{h}\right\|_{L^{2}\left(K_{\delta_{j}}\right)} \leq \frac{\Lambda}{\lambda}\left\|\nabla v_{l i n, K_{j}}^{H}\right\|_{L^{2}\left(K_{\delta_{j}}\right)} .
$$

For a proof, see [6, Lemma 3]).

The next lemma is fundamental for proving the stability of the DG-HMM.

Lemma 4.3. Let $v^{H}, w^{H} \in V_{H}\left(\Omega, \mathcal{T}_{H}\right)$ and let $v_{K}^{h}, K \in \mathcal{T}_{H}$ be the solutions of (2.6) constrained by $v^{H}$, with boundary conditions given by (2.9) or (2.10). Assume that (2.2) holds. Then,

$$
\int_{\Gamma}\left\{\overline{a^{\varepsilon} \nabla v^{h}}\right\} \llbracket w^{H} \rrbracket d s \leq C \alpha^{-1 / 2}\left\|\nabla v^{H}\right\|_{L^{2}(\Omega)}\left|w^{H}\right|_{*},
$$

where $\alpha$ is the penalty parameter (see (3.10) $), \int_{\Gamma}=\sum_{\mathcal{E}} \int_{e}$, and where the constant $C$ is independent of $H, h$ and $\varepsilon$. 
Proof. Applying the Cauchy-Schwarz inequality gives

$$
\sum_{\mathcal{E}} \int_{e}\left(\left\{\overline{a^{\varepsilon} \nabla v^{h}}\right\} \llbracket w^{H} \rrbracket d s \leq \alpha^{-1 / 2}\left(\sum_{\mathcal{E}} H_{e}\left\|\left\{\overline{a^{\varepsilon} \nabla v^{h}}\right\}\right\|_{L^{2}(e)}^{2}\right)^{1 / 2}\left|w^{H}\right|_{*} .\right.
$$

Let $e \in \mathcal{E}_{\text {int }}$. We have

$$
\begin{aligned}
& \left\|\left\{\overline{a^{\varepsilon} \nabla v^{h}}\right\}\right\|_{L^{2}(e)}^{2}=\sum_{i=1}^{d} \frac{1}{4} \int_{e}\left|\frac{1}{\left|K_{\delta}^{+}\right|} \int_{K_{\delta}^{+}}\left(a^{\varepsilon} \nabla v_{K^{+}}^{h}\right)^{i} d x+\frac{1}{\left|K_{\delta}^{-}\right|} \int_{K_{\delta}^{-}}\left(a^{\varepsilon} \nabla v_{K^{-}}^{h}\right)^{i} d x\right|^{2} d s \\
& \leq C \sup _{x \in \Omega}\left\|a^{\varepsilon}\right\|_{F}^{2} \int_{e}\left(\frac{1}{\sqrt{\left|K_{\delta}^{+}\right|}}\left\|\nabla v_{K^{+}}^{h}\right\|_{L^{2}\left(K_{\delta}^{+}\right)}+\frac{1}{\sqrt{\left|K_{\delta}^{-}\right|}}\left\|\nabla v_{K^{-}}^{h}\right\|_{L^{2}\left(K_{\delta}^{-}\right)}\right)^{2} d s \\
& \leq C \int_{e}\left(\left|\nabla v_{+}^{H}\right|+\left|\nabla v_{-}^{H}\right|\right)^{2} d s \leq C \int_{e}\left(\left|\nabla v_{+}^{H}\right|^{2}+\left|\nabla v_{-}^{H}\right|^{2}\right) d s,
\end{aligned}
$$

where $\nabla v_{ \pm}^{H}$ denotes $\left.\nabla v^{H}\right|_{K^{ \pm}}$and where we used the assumption (2.2) and Lemma 4.1. We recall that $\|\cdot\|_{F}$ denotes the Frobenius norm of a given matrix. The case $e \in \mathcal{E}_{B}$ is treated similarly. Summing over $\mathcal{E}$, using the inverse inequality $\int_{\partial K}\left|\nabla v^{H}\right|^{2} d s \leq C H_{K}^{-1} \int_{K}\left|\nabla v^{H}\right|^{2} d x$, where $C$ depends on the shape regularity and the dimension $d$ and $H_{K}$ denotes the diameter of the triangle $K$, we obtain

$$
\begin{aligned}
& \sum_{e \in \mathcal{E}} H_{e}\left\|\left\{\overline{a^{\varepsilon} \nabla v^{h}}\right\}\right\|_{L^{2}(e)}^{2} d s \leq C \sum_{e \in \mathcal{E}} \int_{e} H_{e}\left(\left|\nabla v_{+}^{H}\right|^{2}+\left|\nabla v_{-}^{H}\right|^{2}\right) d s \\
& \leq C \sum_{K \in \mathcal{T}_{H}} H_{K} \int_{\partial K}\left|\nabla v^{H}\right|^{2} d s \leq C \sum_{K \in \mathcal{T}_{H}} \int_{K}\left|\nabla v^{H}\right|^{2} d x=C\left\|\nabla v^{H}\right\|_{L^{2}(\Omega)}^{2},
\end{aligned}
$$

and the result follows.

We can now prove the existence and uniqueness of the solution of problem (3.11).

Theorem 4.4. Assume that (2.2) holds. Then there exists a threshold value $\alpha_{\text {min }}$ for the penalty parameter depending only on the shape regularity of the triangulation $\mathcal{T}_{H}, d, \ell$ and the bound in (2.2), such that for $\alpha \geq \alpha_{\text {min }}$ the bilinear form (3.10) is uniformly elliptic and bounded on $V_{H}\left(\Omega, \mathcal{T}_{H}\right) \times V_{H}\left(\Omega, \mathcal{T}_{H}\right)$ and the problem (3.11) has a unique solution in $u^{H} \in V_{H}\left(\Omega, \mathcal{T}_{H}\right)$ which satisfies

$$
\left\|u^{H}\right\| \leq C\|f\|_{L^{2}(\Omega)},
$$

where $C$ is independent of $H, h$, and $\varepsilon$. 
Proof. To show the uniform ellipticity of (3.10) we use (2.2), Lemmas 4.1 and 4.3 , and compute

$$
\begin{aligned}
& B_{D G}\left(v^{H}, v^{H}\right)=\sum_{K \in \mathcal{T}_{H}} \frac{|K|}{\left|K_{\delta}\right|} \int_{K_{\delta}} a^{\varepsilon}(x) \nabla v_{K}^{h} \cdot \nabla v_{K}^{h} d x d x \\
& -2 \int_{\Gamma}\left\{\overline{\left(a^{\varepsilon} \nabla v^{h}\right)}\right\} \llbracket v^{H} \rrbracket d s+\int_{\Gamma} \mu \llbracket v^{H} \rrbracket^{2} d s \\
& \geq \lambda \sum_{K \in \mathcal{T}_{H}}\left\|\nabla v^{H}\right\|_{L^{2}(K)}^{2}-2 C \alpha^{-1 / 2}\left\|\nabla v^{H}\right\|_{L^{2}(\Omega)}\left|v^{H}\right|_{*}+\sum_{e \in \mathcal{E}} \int_{e}\left\|\mu^{1 / 2} \llbracket v^{H} \rrbracket\right\|_{L^{2}(e)}^{2} \\
& \geq \min (\lambda, 1)\left(\sum_{K \in \mathcal{T}_{H}}\left\|\nabla v^{H}\right\|_{L^{2}(K)}^{2}+\sum_{e \in \mathcal{E}} \int_{e}\left\|\mu^{1 / 2} \llbracket v^{H} \rrbracket\right\|_{L^{2}(e)}^{2}\right) \\
& -2 C \alpha^{-1 / 2}\left(\sum_{K \in \mathcal{T}_{H}}\left\|\nabla v^{H}\right\|_{L^{2}(K)}^{2}\right)^{1 / 2}\left(\sum_{e \in \mathcal{E}} \int_{e}\left\|\mu^{1 / 2} \llbracket v^{H} \rrbracket\right\|_{L^{2}(e)}^{2}\right)^{1 / 2} \\
& \geq\left(\min (\lambda, 1)-C \alpha^{-1 / 2}\right)\left(\sum_{K \in \mathcal{T}_{H}}\left\|\nabla v^{H}\right\|_{L^{2}(K)}^{2}+\sum_{e \in \mathcal{E}} \int_{e}\left\|\mu^{1 / 2} \llbracket v^{H} \rrbracket\right\|_{L^{2}(e)}^{2}\right) \\
& =\left(\min (\lambda, 1)-C \alpha^{-1 / 2}\right)\left\|v^{H}\right\|^{2} .
\end{aligned}
$$

This proves that the bilinear form (3.10) is uniformly elliptic, provided $\alpha$ is large enough. We next show that (3.10) is bounded (independently of $H, h$ and $\varepsilon$ ). For that, we use again Lemmas 4.1 and 4.3 , the Cauchy-Schwarz inequality and (2.2). We obtain

$$
\begin{aligned}
\left|B_{D G}\left(v^{H}, w^{H}\right)\right| \leq & \tilde{C} \sum_{K \in \mathcal{T}_{H}}\left\|\nabla v^{H}\right\|_{L^{2}(K)}\left\|\nabla w^{H}\right\|_{L^{2}(K)}+\bar{C} \alpha^{-1 / 2}\left\|\nabla v^{H}\right\|_{L^{2}(\Omega)}\left|w^{H}\right|_{*} \\
& +\bar{C} \alpha^{-1 / 2}\left\|\nabla w^{H}\right\|_{L^{2}(\Omega)}\left|v^{H}\right|_{*}+\left|v^{H}\right|_{*}\left|w^{H}\right|_{*} \\
\leq & \max \left(1, \tilde{C}, \bar{C} \alpha^{-1 / 2}\right)\left(\left\|\nabla v^{H}\right\|_{L^{2}(\Omega)}\left\|\nabla w^{H}\right\|_{L^{2}(\Omega)}\right. \\
& \left.+\left\|\nabla w^{H}\right\|_{L^{2}(\Omega)}\left|v^{H}\right|_{*}+\left\|\nabla v^{H}\right\|_{L^{2}(\Omega)}\left|w^{H}\right|_{*}+\left|v^{H}\right|_{*}\left|w^{H}\right|_{*}\right) \\
\leq & C\|v\|\|w\| .
\end{aligned}
$$

The existence and the uniqueness of the solution of (3.11) and the estimates (4.9) now follow from the Lax-Milgram theorem.

Remark 4.5. Notice that we have not specified the order of the micro FE method $S^{q}\left(K_{\delta}, \mathcal{T}_{h}\right)$ used to solve the cell problem (2.6) in the bilinear form (3.10). We emphasize that the results of Theorem 4.4 are valid for any $q \geq 1$.

\section{A PRIORI ERROR ESTIMATES}

In this section we shall derive a priori error estimates for the DG-HMM. We perform the analysis in two successive steps. In a first step (Section 5.2) we analyze the contribution to the error coming from the macro and micromeshes (recall that our DG-HMM approximation relies on macro and micro FE spaces). In a second step (Section 5.3) we analyze the so-called modeling error, i.e., the contribution to the error which remains when macro and micro meshsizes tend to zero. 
5.1. Preliminary results. We collect in this section several useful preliminary results. We start by defining a numerically homogenized tensor which will be used in the a priori analysis. Consider the Sobolev space $W\left(K_{\delta}\right)$ defined in (2.10) or (2.9) and the FE space $S^{q}\left(K_{\delta}, \mathcal{T}_{h}\right) \subset W\left(K_{\delta}\right)$ defined in (2.8). For each vector $\mathbf{e}_{\mathbf{i}}, i=1, \ldots, d$ of the canonical basis of $\mathbb{R}^{d}$ let $\psi_{K}^{i, h} \in S^{q}\left(K_{\delta}, \mathcal{T}_{h}\right)$ be the solution of the micro problem (2.6) with modified right-hand side

$$
\int_{K_{\delta}} a^{\varepsilon}(x) \nabla \psi_{K}^{i, h} \cdot \nabla z^{h} d x=-\int_{K_{\delta}} a^{\varepsilon}(x) \mathbf{e}_{\mathbf{i}} \cdot \nabla z^{h} d x, \forall z^{h} \in S^{q}\left(K_{\delta}, \mathcal{T}_{h}\right) .
$$

We also consider the problem: find $\psi_{K}^{i} \in W\left(K_{\delta}\right)$ such that

$$
\int_{K_{\delta}} a^{\varepsilon}(x) \nabla \psi_{K}^{i} \cdot \nabla z d x=-\int_{K_{\delta}} a^{\varepsilon}(x) \mathbf{e}_{\mathbf{i}} \cdot \nabla z d x, \forall z \in W\left(K_{\delta}\right) .
$$

For the analysis of the micro problems, some regularity of the oscillating tensor is required. As we use standard a priori results of FEMs in the sampling domain, we will need (local) $H^{2}$ regularity. However, the coefficient $a^{\varepsilon}$ is allowed to be discontinuous (at the macroscopic level) through smooth interfaces. We assume that the macromesh (i.e., the interface between two neighboring elements) is aligned with these discontinuities.

(H1) $\left.a^{\varepsilon}\right|_{K} \in W^{1, \infty}(K), \forall K \in \mathcal{T}_{H}$ and $\left|a_{i j}^{\varepsilon}\right|_{W^{1, \infty}(K)} \leq C_{K} \varepsilon^{-1}$ for $i, j=1, \ldots, d$.

In the analysis we will often use a constant $C=\max _{K} C_{K}$ independent of $K$. It is clear that if (H1) is valid for an initial mesh assumed to be aligned with the possible discontinuities of $a^{\varepsilon}$, it is still valid (with the same value of $C$ ) for every mesh obtained by refining the initial one.

Remark 5.1. Without any further knowledge about the structure of the oscillating tensor $a^{\varepsilon}$, we will impose Dirichlet boundary conditions for (2.6) (or (5.1), (5.2)). Assuming (H1) one can show $\left|\psi_{K}^{i}\right|_{H^{2}\left(K_{\delta}\right)} \leq C \varepsilon^{-1} \sqrt{\left|K_{\delta}\right|}$, with $C$ independent of $\varepsilon$ and the domain $K_{\delta}$ (this follows from classical $H^{2}$ regularity results [11, Chap. 2.6, eq. (6.23)]).

If $a^{\varepsilon}=a(x, x / \varepsilon)=a(x, y)$ is $y$-periodic in a cube $Y$ (e.g., $\left.Y=[0,1]^{d}\right)$ and $\delta / \varepsilon \in \mathbb{N}$, then assuming $(\mathbf{H 1})$ one can show $\left|\psi_{K}^{i}\right|_{H^{2}\left(K_{\delta}\right)} \leq C \varepsilon^{-1} \sqrt{\left|K_{\delta}\right|}$, for periodic boundary conditions and slow variable collocated in each sampling domain $K_{\delta_{j}}$ at the quadrature points $x_{K_{j}}$ in (5.1), (5.2) (this follows classical regularity results for solutions of periodic boundary value problems; see [21, Chap. $3]$. In the locally periodic case, with fixed slow variable, higher order estimates $\left|\psi_{K}^{i}\right|_{H^{q+1}\left(K_{\delta}\right)} \leq C \varepsilon^{-q} \sqrt{\left|K_{\delta}\right|}, q \in \mathbb{N}$ can also be obtained provided there is appropriate regularity of the oscillating tensor.

We then define two tensors:

$$
a_{K}^{0}=\frac{1}{\left|K_{\delta}\right|} \int_{K_{\delta}} a^{\varepsilon}(x)\left(I+J_{\psi_{K}^{h}(x)}^{T}\right) d x
$$

where $J_{\psi_{K}^{h}(x)}$ is a $d \times d$ matrix with entries $\left(J_{\psi_{K}^{h}(x)}\right)_{i \ell}=\left(\partial \psi_{K}^{i, h}\right) /\left(\partial x_{\ell}\right)$, and

$$
\bar{a}_{K}^{0}=\frac{1}{\left|K_{\delta}\right|} \int_{K_{\delta}} a^{\varepsilon}(x)\left(I+J_{\psi_{K}(x)}^{T}\right) d x
$$

where $J_{\psi_{K}(x)}$ is a $d \times d$ matrix with entries $\left(J_{\psi_{K}(x)}\right)_{i \ell}=\left(\partial \psi_{K}^{i}\right) /\left(\partial x_{\ell}\right)$. We emphasize that the tensor (5.3) is never computed explicitly in the DG-HMM and will only be used as a tool for analysis. 
The following lemma quantifies the so-called "micro-error" and was first derived in [3. Recall that the measure of the sampling domain $K_{\delta}$ is given by $\left|K_{\delta}\right|=\delta^{d}$.

Lemma 5.2. Let $\psi_{K}^{i}, \psi_{K}^{i, h}$ be the solutions of (5.1) and (5.2), respectively. For general tensor $a^{\varepsilon}(x)$ we assume that Dirichlet boundary conditions are used in (5.1) and (5.2). If $a^{\varepsilon}(x)=a\left(x_{K}, x / \varepsilon\right)=a\left(x_{K}, y\right)$ is $y$-periodic in $Y$, collocated in the slow variables at the quadrature points of the sampling domain $K_{\delta_{j}}$ and $\delta / \varepsilon \in \mathbb{N}$, we assume that either Dirichlet and periodic boundary conditions are used in (5.1) and (5.2). Consider the FE space (2.8) with $q=1$ and assume that (2.2) and (H1) hold. Then

$$
\sup _{K \in \mathcal{T}_{H}}\left\|\bar{a}_{K}^{0}-a_{K}^{0}\right\|_{F} \leq C\left(\frac{h}{\varepsilon}\right)^{2},
$$

where $C$ is independent of $h$ and $\varepsilon$.

Proof. We first define the functions $\varphi_{K}^{i}:=x_{i}+\psi_{K}^{i}, \psi_{K}^{i} \in W\left(K_{\delta}\right)$ and $\varphi_{K}^{i, h}:=x_{i}+$ $\psi_{K}^{i, h}, \psi_{K}^{i, h} \in S^{1}\left(K_{\delta}, \mathcal{T}_{h}\right)$, and observe in view of (5.1), (5.2) that $\int_{K_{\delta}} a^{\varepsilon}(x) \nabla\left(\varphi_{K}^{j, h}-\right.$ $\left.\varphi_{K}^{j}\right) \cdot \nabla z^{h}=0$, for all $z^{h} \in S^{1}\left(K_{\delta}, \mathcal{T}_{h}\right)$. We then obtain

$$
\begin{aligned}
\left|\left(\bar{a}_{K}^{0}\right)_{i j}-\left(a_{K}^{0}\right)_{i j}\right| & =\left|\frac{1}{\left|K_{\delta}\right|} \int_{K_{\delta}}\left(a^{\varepsilon}(x) \nabla \varphi_{K}^{j} \cdot \nabla \varphi_{K}^{i}-a(x)^{\varepsilon} \nabla \varphi_{K}^{j, h} \cdot \nabla \varphi_{K}^{i, h}\right) d x\right| \\
& =\left|\frac{1}{\left|K_{\delta}\right|} \int_{K_{\delta}} a^{\varepsilon}(x) \nabla\left(\varphi_{K}^{j, h}-\varphi_{K}^{j}\right) \cdot \nabla\left(\varphi_{K}^{i, h}-\varphi_{K}^{i}\right) d x\right| \\
& \leq\left. C\left|\frac{1}{\left|K_{\delta}\right|} \| \nabla\left(\psi_{K}^{j, h}-\psi_{K}^{j}\right)\right|\right|_{L^{2}\left(K_{\delta}\right)} \cdot|| \nabla\left(\psi_{K}^{i, h}-\psi_{K}^{i}\right)||_{L^{2}\left(K_{\delta}\right)} \mid \\
& \leq C \frac{1}{\left|K_{\delta}\right|} h^{2}\left|\psi_{K}^{j}\right|_{H^{2}\left(K_{\delta}\right)} \cdot\left|\psi_{K}^{i}\right|_{H^{2}\left(K_{\delta}\right)},
\end{aligned}
$$

where we used the symmetry of $a^{\varepsilon}$ and the standard FE estimate [23, Thm. 3.2.2] in the last inequality. Using (H1), the hypothesis of the lemma on the boundary conditions and Remark 5.1 yields

$$
\left|\psi_{K}^{j}\right|_{H^{2}\left(K_{\delta}\right)} \leq C\left|K_{\delta}\right|^{1 / 2} \varepsilon^{-1}
$$

where $C$ is independent of $K_{\delta}$ and $\varepsilon$. Inserting this inequality in (15.6) gives the result.

Provided higher order regularity of the solution of problem (5.2) (see Remark 5.1) we obtain the following corollary.

Corollary 5.3. In addition to (2.2) assume that $\psi_{K}^{i}$, the solution of (5.2) (with either periodic or Dirichlet boundary conditions), satisfies

$$
\left|\psi_{K}^{i}\right|_{H^{q+1}\left(K_{\delta}\right)} \leq C \varepsilon^{-q} \sqrt{\left|K_{\delta}\right|}
$$

for a given $q \in \mathbb{N}$ with $C$ independent of $\varepsilon$ and the domain $K_{\delta}$, then by solving (5.1) in $S^{q}\left(K_{\delta}, \mathcal{T}_{h}\right)$ we obtain

$$
\sup _{K \in \mathcal{T}_{H}}\left\|\bar{a}_{K}^{0}-a_{K}^{0}\right\|_{F} \leq C\left(\frac{h}{\varepsilon}\right)^{2 q} .
$$

Proof. Follows the lines of Lemma 5.2 using (5.8) instead of (5.7). 
Notice that the expression $\hat{h}=h / \varepsilon$ is independent of $\varepsilon$. Indeed, if we use $N_{\text {micro }}$ elements in each space dimension for the discretization of the sampling domains, we have $h=\delta / N_{\text {micro }}$ and therefore $\hat{h}=(\delta / \varepsilon) \cdot\left(1 / N_{\text {micro }}\right)$. Since $\delta$ scales with $\varepsilon$, typically $\delta=C \varepsilon$ (with a constant $C$ of moderate size), we have $\hat{h}=\left(C / N_{\text {micro }}\right)$.

The following two lemmas allow us to give a reformulation of the DG-HMM.

Lemma 5.4. Let $v_{K}^{h}, w_{K}^{h}$ be the solutions of (2.6) constrained by $v^{H}, w^{H} \in$ $V_{H}\left(\Omega, \mathcal{T}_{H}\right)$ with boundary conditions given by (2.9) or (2.10). Then

$$
\frac{1}{\left|K_{\delta}\right|} \int_{K_{\delta}} a^{\varepsilon}(x) \nabla v_{K}^{h} \cdot \nabla w_{K}^{h} d x=\frac{1}{|K|} \int_{K} a_{K}^{0} \nabla v^{H} \cdot \nabla w^{H} d x .
$$

Proof. The proof is similar to (A.1) in [9, App. A] (see also formula (63) in 6]). We first notice that the solution $v_{K}^{h}$ or $w_{K}^{h}$ of the micro problem (2.6) can be written as

$$
v_{K}^{h}(x)=v^{H}(x)+\sum_{i=1}^{d} \psi_{K}^{i, h}(x) \frac{\partial v^{H}(x)}{\partial x_{i}},
$$

where the functions $\psi_{K}^{i, h}$ are defined above. Using this formula and noting that $v_{K}^{h}$ and $w_{K}^{h}$ solve the problem (2.6), and that $\nabla v^{H}, \nabla w^{H}$ are constant, we see that

$$
\begin{aligned}
& \frac{1}{\left|K_{\delta}\right|} \int_{K_{\delta}} a^{\varepsilon}(x) \nabla v_{K}^{h} \cdot \nabla w_{K}^{h} d x \\
= & \frac{1}{\left|K_{\delta}\right|} \int_{K_{\delta}} a^{\varepsilon}(x)\left(\nabla v^{H}+\sum_{i=1}^{d} \nabla \psi_{K}^{i, h}(x) \frac{\partial v^{H}(x)}{\partial x_{i}}\right) \cdot \nabla w^{H} d x \\
= & \frac{1}{\left|K_{\delta}\right|} \int_{K_{\delta}} a^{\varepsilon}(x)\left(I+J_{\psi_{K}^{h}(x)}^{T}\right) \nabla v^{H} \cdot \nabla w^{H} d x, \\
= & \frac{1}{|K|} \int_{K} a_{K}^{0} \nabla v^{H} \cdot \nabla w^{H} d x,
\end{aligned}
$$

where we used the definition (5.3).

Corollary 5.5. Let $v_{K}^{h}$ be the solution of (2.6) constrained by $v^{H} \in V_{H}\left(\Omega, \mathcal{T}_{H}\right)$ with boundary conditions given by (2.9) or (2.10). Then

$$
\frac{1}{\left|K_{\delta}\right|} \int_{K_{\delta}} a^{\varepsilon}(x) \nabla v_{K}^{h} d x=\frac{1}{|K|} \int_{K} a_{K}^{0} \nabla v^{H} d x=a_{K}^{0} \nabla v^{H} .
$$

Proof. Follows the lines of Lemma 5.4

Lemma 5.6. Let $K^{+}, K^{-} \in \mathcal{T}_{H}$ having a common interface e. Let $v_{K^{ \pm}}^{h}$ be the solution of (2.6) in $K^{+}, K^{-}$, respectively, constrained by $v^{H} \in V_{H}^{1}\left(\Omega, \mathcal{T}_{H}\right)$ with boundary conditions given by (2.9) or (2.10). Then,

$$
\left\|\left\{\overline{a^{\varepsilon} \nabla v^{h}}\right\}\right\|_{L^{2}(e)}=\left\|\left\{a_{K}^{0} \nabla v^{H}\right\}\right\|_{L^{2}(e)} .
$$

Proof. We have

$$
\left\|\left\{\overline{a^{\varepsilon} \nabla v^{h}}\right\}\right\|_{L^{2}(e)}^{2}=\frac{1}{4} \int_{e}\left|\frac{1}{\left|K_{\delta}^{+}\right|} \int_{K_{\delta}^{+}}\left(a^{\varepsilon} \nabla v_{K^{+}}^{h}\right) d x+\frac{1}{\left|K_{\delta}^{-}\right|} \int_{K_{\delta}^{-}}\left(a^{\varepsilon} \nabla v_{K^{-}}^{h}\right) d x\right|^{2} d s .
$$


Inserting $\nabla v_{K^{ \pm}}^{h}=\nabla v_{ \pm}^{H}+\sum_{i=1}^{d} \nabla \psi_{K^{ \pm}}^{i, h}(x) \frac{\partial v_{ \pm}^{H}(x)}{\partial x_{i}}$ and using Corollary 5.5 gives

$$
\left\|\left\{\overline{a^{\varepsilon} \nabla v^{h}}\right\}\right\|_{L^{2}(e)}^{2}=\frac{1}{4} \int_{e}\left|a_{K^{+}}^{0} \nabla v_{+}^{H}+a_{K^{-}}^{0} \nabla v_{-}^{H}\right|^{2} d s,
$$

where $v_{ \pm}^{H}=\left.v^{H}\right|_{K^{ \pm}}$. Taking the square root of the right-hand side of the above equality gives (5.12).

The reformulation of the DG-HMM (3.10) read as follows.

Proposition 5.7. Let $v^{H}, w^{H} \in V_{H}\left(\Omega, \mathcal{T}_{H}\right) \times V_{H}\left(\Omega, \mathcal{T}_{H}\right)$. Then (3.10) can be written as

$$
\begin{aligned}
B_{D G}\left(v^{H}, w^{H}\right) & =\sum_{K \in \mathcal{T}_{H}} \int_{K} a_{K}^{0} \nabla v^{H} \cdot \nabla w^{H} d x-\int_{\Gamma}\left(\left\{a_{K}^{0} \nabla v^{H}\right\} \llbracket w^{H} \rrbracket\right. \\
& \left.+\left\{a_{K}^{0} \nabla w^{H}\right\} \llbracket v^{H} \rrbracket\right) d s+\int_{\Gamma} \mu \llbracket v^{H} \rrbracket \llbracket w^{H} \rrbracket d s .
\end{aligned}
$$

Proof. Follows for Lemmas 5.4 and 5.6 .

Standard IP discontinuous Galerkin for the homogenized problem. Consider the homogenized problem (2.3). We note that the error analysis of the macro FE-HMM (see [3], 6], 32]) relies on convergence rates for FEM with numerical integration 23 . While well documented for FEM, the effect of numerical quadrature in DG-FEM has, to the best of our knowledge, not been analyzed for elliptic problems with variable coefficients. Such results (for single scale elliptic problems) have yet to be derived. As this is not the focus of our paper and to avoid dealing with numerical quadrature for standard DG-FEM, we assume the following structure assumption on the oscillating tensor

(H2) $a^{\varepsilon}=a(x, x / \varepsilon)=a(x, y)$ is $Y$-periodic in $y$, and for $\left.K \in \mathcal{T}_{H} a(\cdot, y)\right|_{K}$ is constant, where $Y=(0,1)^{d}$. A consequence of $(\mathbf{H 2})$ is that the homogenized tensor $a^{0}$ will be piecewise constant in any $K \in \mathcal{T}_{H}$ (this follows from classical periodic homogenization see e.g., [24]). We note that an assumption as (H2) has been been used by many authors for the analysis of multiscale method [15, 35], 36], 45. We emphasize that results derived for single scale DG-FEM with numerical integration could be readily used in the analysis developed in this paper and the above assumption could be relaxed to standard local periodicity, i.e, $a(x, y)$ is $Y$ periodic in $y$ and smooth in $x$. Smoothness of the homogenized tensor in $K$ will still be needed to have a well-defined trace for $a^{0}$ when using standard DG-FEM as, e.g., the requirement for $a_{i j}^{0}(x)$ to be Lipschitz continuous inside any $K \in \mathcal{T}_{H}$. The local periodicity assumption is also essential for the analysis of the FE-HMM [3], 32.

We now define for $v^{H}, w^{H} \in V_{H}\left(\Omega, \mathcal{T}_{H}\right) \times V_{H}\left(\Omega, \mathcal{T}_{H}\right)$ the usual IP discontinuous Galerkin method with piecewise constant coefficients in each $K \in \mathcal{T}_{H}$ denoted by $a^{0}\left(x_{K}\right)$ (recall that $x_{K}$ is the quadrature node in $K$ ).

$$
\begin{aligned}
B_{D G, 0}\left(v^{H}, w^{H}\right) & =\sum_{K \in \mathcal{T}_{H}} \int_{K} a^{0}\left(x_{K}\right) \nabla v^{H} \cdot \nabla w^{H} d x-\int_{\Gamma}\left(\left\{a^{0} \nabla v^{H}\right\} \llbracket w^{H} \rrbracket\right. \\
& \left.+\left\{a^{0} \nabla w^{H}\right\} \llbracket v^{H} \rrbracket\right) d s+\int_{\Gamma} \mu \llbracket v^{H} \rrbracket \llbracket w^{H} \rrbracket
\end{aligned}
$$


where for an interface $e$ of $K^{+}, K^{-}$, we have

$$
\left\{a^{0} \nabla v^{H}\right\}=\frac{1}{2}\left(a^{0}\left(x_{K^{+}}\right) \nabla v^{H}\left(x_{K^{+}}\right)+a^{0}\left(x_{K^{-}}\right) \nabla v^{H}\left(x_{K^{-}}\right)\right) .
$$

We notice that with our assumption on $a^{0}$ (induced by $(\mathbf{H 2})$ ), the above bilinear form is well defined on $V(H) \times V(H)$. The coercivity of (5.14) in $V_{H}\left(\Omega, \mathcal{T}_{H}\right) \times$ $V_{H}\left(\Omega, \mathcal{T}_{H}\right)$ and the boundedness on $V(H) \times V(H)$ can be established following the results in [17]. We denote by $u^{0, H}$ the solution of the problem

$$
B_{D G, 0}\left(u^{0, H}, v^{H}\right)=\int_{\Omega} f v^{H} d x, \forall v^{H} \in V_{H}\left(\Omega, \mathcal{T}_{H}\right) .
$$

5.2. A-priori estimates: macro an micro errors. In this section we derive our main results: an optimal a priori convergence rate for the DG-HMM in the $\|\cdot\| \|$ and the $L^{2}$ norm. Following the framework of the error analysis for the FE-HMM 6] we decompose the error in

$$
\left\|u^{0}-u^{H}\left|\|\leq\| u^{0}-u^{0, H}\|\mid+\| u^{0, H}-u^{H} \|,\right.\right.
$$

where $u^{0}$ is the solution of (2.3),$u^{0, H}$ is the solution of (5.15) and $u^{H}$ is the solution of (3.11).

The first term on the right-hand side of (5.16) is the macro error and is related to the macro solver and the error induced by the macroscopic triangulation. This error can be estimated in a standard way (see below). The second term in the right-hand side of (5.16) will further be decomposed in modeling and micro errors (see (5.21) $)$. The micro error is related to the error introduced by solving (2.6) in a micro FE space while the modeling error quantifies how well the coarse graining procedure captures the effective solution of the multiscale problem (see Section 5.3 ).

Macro error. The term $\left\|u^{0}-u^{0, H}\right\| \mid$ can be estimated following classical a priori error analysis for IP discontinuous Galerkin method applied to (2.3). The convergence rates read (see [17]):

Lemma 5.8. Let $u^{0}, u^{0, H}$ be the solutions of (2.3) and (5.15), respectively. Assume that (2.2) and (H2) hold, and that $u^{0} \in H^{2}(\Omega)$. Then

$$
\begin{aligned}
& \left\|u^{0}-u^{0, H}\right\| \leq C H, \\
& \left\|u^{0}-u^{0, H}\right\|_{L^{2}(\Omega)} \leq C H^{2} .
\end{aligned}
$$

Remark 5.9. It is well known that with piecewise discontinuous coefficients the solution $u^{0}$ of (2.3) may not be in $H^{2}(\Omega)$ and the above convergence rate may deteriorate (see [30] for convergence results for the IP DG-FEM with discontinuous coefficients). This possible deterioration of the convergence rate is an issue for standard FEM (or DG-FEM) applied to (the single scale) problem (2.3) and does not constitute a limitation specific to our multiscale strategy.

Micro error. We start with a Strang type lemma. From

$$
\begin{aligned}
C\left\|u^{0, H}-u^{H}\right\|^{2} & \leq B_{D G, 0}\left(u^{0, H}-u^{H}, u^{0, H}-u^{H}\right) \\
& =B_{D G}\left(u^{H}, u^{0, H}-u^{H}\right)-B_{D G, 0}\left(u^{H}, u^{0, H}-u^{H}\right),
\end{aligned}
$$

we deduce that

$$
\left\|u^{0, H}-u^{H}\right\| \leq C \sup _{v^{H} \in V_{H}\left(\Omega, \mathcal{T}_{H}\right)} \frac{\left|B_{D G}\left(u^{H}, v^{H}\right)-B_{D G, 0}\left(u^{H}, v^{H}\right)\right|}{\left\|v^{H}\right\|} .
$$


Lemma 5.10. Let $u^{H}$ and $u^{0, H}$ be the solutions of (3.11) and (5.15), respectively. Assume that (2.2) and (H2) hold. Then we have

$$
\left\|u^{0, H}-u^{H}\right\| \leq C \sup _{K \in \mathcal{T}_{H}}\left\|a^{0}\left(x_{K}\right)-a_{K}^{0}\right\|_{F}\left\|u^{H}\right\|,
$$

where the constant $C$ is independent of $H, h$ and $\varepsilon$.

Proof. We have

$$
\begin{gathered}
\left|B_{D G}\left(u^{H}, v^{H}\right)-B_{D G, 0}\left(u^{H}, v^{H}\right)\right| \leq \underbrace{\left|\sum_{K \in \mathcal{T}_{H}} \int_{K}\left(a^{0}\left(x_{K}\right)-a_{K}^{0}\right) \nabla u^{H} \cdot \nabla v^{H} d x\right|}_{I_{1}} \\
+\underbrace{\left|\int_{\Gamma}\left(\left\{\left(a^{0}\left(x_{K}\right)-a_{K}^{0}\right) \nabla u^{H}\right\} \llbracket v^{H} \rrbracket+\left\{\left(a^{0}\left(x_{K}\right)-a_{K}^{0}\right) \nabla v^{H}\right\} \llbracket u^{H} \rrbracket\right) d s\right|}_{I_{2}} .
\end{gathered}
$$

The expression $I_{1}$ can be bounded as

$$
I_{1} \leq \sup _{K \in \mathcal{T}_{H}}\left\|a^{0}\left(x_{K}\right)-a_{K}^{0}\right\|_{F}\left\|\nabla u^{H}\right\|_{L^{2}(\Omega)}\left\|\nabla v^{H}\right\|_{L^{2}(\Omega)} .
$$

Following the proof of the Lemma 4.3 we can estimate the expression $I_{2}$ as

$$
I_{2} \leq C \alpha^{-1 / 2} \sup _{K \in \mathcal{T}_{H}}\left\|a^{0}\left(x_{K}\right)-a_{K}^{0}\right\|_{F}\left\|u^{H}\right\|\left\|v^{H}\right\| .
$$

Using the above estimates and (5.19) gives the result.

In view of (5.20) we consider the decomposition,

$$
\sup _{K \in \mathcal{T}_{H}}\left\|a^{0}\left(x_{K}\right)-a_{K}^{0}\right\|_{F} \leq \underbrace{\sup _{K \in \mathcal{T}_{H}}\left\|a^{0}\left(x_{K}\right)-\bar{a}_{K}^{0}\right\|_{F}}_{\text {err mod }}+\underbrace{\sup _{K \in \mathcal{T}_{H}}\left\|\bar{a}_{K}^{0}-a_{K}^{0}\right\|_{F}}_{\text {err }_{\text {mic }}},
$$

where $\bar{a}_{K}^{0}$ is defined in (5.4) and corresponds to a tensor similar to $a_{K}^{0}$ but using exact micro functions. Hence, the second term of the right-hand side of (5.21) corresponds to the error introduced by the micro FEM and the first term measures how accurate our modeling of the micro problems is when compared to the homogenized tensor. The micro error has been estimated in Lemma 5.2 and reads

$$
\sup _{K \in \mathcal{T}_{H}}\left\|\bar{a}_{K}^{0}-a_{K}^{0}\right\|_{F} \leq C\left(\frac{h}{\varepsilon}\right)^{2}
$$

We can now state our first a priori result. As for Lemma 5.2 we assume in the following theorem that periodic or Dirichlet boundary conditions are used for the micro problems (2.6) if the locally periodic tensor $a^{\varepsilon}$ is such that $\delta / \varepsilon \in \mathbb{N}$, and that Dirichlet boundary conditions are used otherwise. 
Theorem 5.11. Let $u^{0}, u^{H}$ be the solutions of (2.3) and (3.11), respectively. Assume that (2.2), (H1) and (H2) hold, and that $u^{0} \in H^{2}(\Omega)$. Then 3

$$
\begin{array}{r}
\left\|u^{0}-u^{H}\right\| \leq C\left(H+\left(\frac{h}{\varepsilon}\right)^{2}+\sup _{K \in \mathcal{T}_{H}}\left\|a^{0}\left(x_{K}\right)-\bar{a}_{K}^{0}\right\|_{F}\right), \\
\left\|u^{0}-u^{H}\right\|_{L^{2}(\Omega)} \leq C\left(H^{2}+\left(\frac{h}{\varepsilon}\right)^{2}+\sup _{K \in \mathcal{T}_{H}}\left\|a^{0}\left(x_{K}\right)-\bar{a}_{K}^{0}\right\|_{F}\right),
\end{array}
$$

where $C$ is independent of $H, h$ and $\varepsilon$.

Proof. Follows from the decomposition (5.16), Lemmas 5.2, 5.8 and 5.10,

Corollary 5.12. Assume in addition to the hypothesis of Theorem 5.11 that (5.8) hold, then we can replace the terms $\left(\frac{h}{\varepsilon}\right)^{2}$ in the above theorem by $\left(\frac{h}{\varepsilon}\right)^{2 q}$.

Complexity. The convergence rates derived in Theorem 5.11 allow us to study the complexity of the DG-HMM. First, we notice that $\operatorname{err}_{\text {mod }}=\sup _{K \in \mathcal{T}_{H}} \| a^{0}\left(x_{K}\right)-$ $\bar{a}_{K}^{0} \|_{F}$ which appears in (5.23) and (5.24) is a quantity independent of the discretization parameters $H, h$ (see Section [5.3). Second, we recall that $\hat{h}=h / \varepsilon$ is independent of $\varepsilon$ and, when using $N_{\text {micro }}$ elements in each space dimension for the discretization of the sampling domains, we have $\hat{h}=\left(C / N_{\text {micro }}\right.$ ) (as $\delta$ scales with $\varepsilon)$. Let us denote by $M_{\text {micro }}$ the number of degrees of freedom (DOF) of a micro FEM in a sampling domain. We have

$$
M_{\text {micro }}=N_{\text {micro }}^{d}=C \hat{h}^{-d} \text {. }
$$

To quantify the complexity of the numerical method, we also define $N_{\text {macro }}$, the number of macroscopic elements in each space dimension, hence, $H=C / N_{\text {macro }}$. Then, the total macroscopic degrees of freedom is given by

$$
M_{\text {macro }}=N_{\text {macro }}^{d}=C H^{-d} \text {. }
$$

Next, we rewrite (5.23) and (5.24) in terms of macro and micro DOF. We obtain

$$
\begin{aligned}
\left\|u^{0}-u^{H}\right\| & \leq C\left(M_{\text {macro }}^{\frac{-1}{d}}+M_{\text {micro }}^{\frac{-2 q}{d}}+\text { err }_{\text {mod }}\right), \\
\left\|u^{0}-u^{H}\right\|_{L^{2}(\Omega)} & \leq C\left(M_{\text {macro }}^{\frac{-2}{d}}+M_{\text {micro }}^{\frac{-2 q}{d}}+\text { err }_{\text {mod }}\right) .
\end{aligned}
$$

In the periodic case, when $\delta / \varepsilon \in \mathbb{N}$, then $e r r_{\text {mod }}=0$ (see Section 5.3) and the above convergence rates show that our method is robust, i.e., converges independently of $\varepsilon$. Assuming that the complexity is proportional to the total number of DOF (e.g., by using a multigrid solver for the linear system) and in view of the error estimates (5.25), (15.26), the total cost of the DG-HMM is $M_{\text {macro }}^{\alpha_{1}} \times \cdot M_{\text {micro }}^{\alpha_{2}}$, where $\alpha_{1}, \alpha_{2}$ depend on the error norm and the type of FEs. In contrast, the memory requirement is only proportional to $M_{\text {macro }}+M_{\text {micro }}$, because the micro problems are independent of one another and thus can be solved one at a time. For example, when $d=2$ and when using piecewise linear FEs for the macro and the micro method we can choose $M_{\text {micro }}=M_{\text {macro }}\left(L^{2}\right.$ norm $)$ and $M_{\text {micro }}=\sqrt{M_{\text {macro }}}\left(H^{1}\right.$ norm), and we have optimal convergence rates with a total of $M_{\text {macro }}^{2}$ and $M_{\text {macro }}^{3 / 2}$ DOF for the $L^{2}$ and the $H^{1}$ norm, respectively.

\footnotetext{
${ }^{3}$ Compared with previous results [11. Thm. 4.5], where linear micro convergence rates were obtained, we see in Theorem 5.11 that we are able to get quadratic micro convergence rates.
} 
We note that, in general, we have superlinear complexity in the macro degrees of freedom for the DG-HMM (as for the FE-HMM). Observe that we have nevertheless achieved a considerable computational saving, as solving the fine scale problem with the standard DG method (3.7) would imply a complexity of $\mathcal{O}\left(\varepsilon^{-d}\right)$. In special cases, for example, when the tensor only depends on a fast variable (e.g., $a^{\varepsilon}=a(x / \varepsilon)$ ), the micro problem can be done once and we have a linear complexity. The issue of the superlinear overall complexity has been addressed for the FEHMM in [10] for locally periodic coefficients, where near optimal computational complexity has been achieved by combining a FEM for the macroscopic solver with a pseudo-spectral method for the micro solver. Such type of micro solvers could also be used for the DG-HMM. We also emphasize that the DG-HMM is trivial to parallelize as the cell problems can be computed independently.

Small scale recovery. The primary goal of the DG-HMM, given the oscillatory problem (2.1), is to approximate the solution of the homogenized or upscaled problem (2.3). While $u^{H}$ converges to the solution $u^{\varepsilon}$ of (2.3) in the $L^{2}$ norm, a convergence of $u^{H}$ to $u^{\varepsilon}$ in the energy or $H^{1}$ norm is not guaranteed in general. Indeed, the oscillations of $u^{\varepsilon}$ introduce $\mathcal{O}(1)$ perturbations in the gradient which are not captured by $u^{H}$. The same behavior holds for the homogenized solution $u^{0}$ of (2.3).

An approximation of the gradient of $u^{\varepsilon}$ can nevertheless be obtained from the known macroscopic numerical solution $u^{H}$ and the known microscopic solutions in the sampling domains computed during the assembly process of the DG-HMM. By extending periodically the known solution $u_{K}^{h}$ in $K_{\delta}$ on $K$ as

$$
u^{p, \varepsilon}(x):=u^{H}(x)+\left(u_{K}^{h}-u^{H}\right)\left(x-[x]_{K_{\delta}}\right) \text { for all } x \in K \in \mathcal{T}_{H},
$$

where for $x \in \mathbb{R}^{d},[x]_{K_{\delta}} \in \delta \mathbb{Z}^{d}$ is such that $x-[x]_{K_{\delta}} \in K_{\delta}$, we obtain a fine scale approximation defined (piecewise) in each macro domain $K$ (see [3], 6] for details). This procedure has first been analyzed for the continuous FE-HMM in [32] (semidiscrete case) and 3 (fully-discrete case). Assuming a smooth locally periodic tensor and periodic boundary conditions for the micro solver (with a sampling domain covering an integer number of the period in each spatial direction) we have

$$
\left\|u^{\varepsilon}-u^{p, \varepsilon}\right\|_{\bar{H}^{1}(\Omega)} \leq C\left(H+\frac{h}{\varepsilon}+\sqrt{\varepsilon}\right),
$$

where $\bar{H}^{1}(\Omega)$ denotes a broken norm $\|u\|_{\bar{H}^{1}(\Omega)}:=\left(\sum_{K \in \mathcal{T}_{H}}\|\nabla u\|_{L^{2}(K)}^{2}\right)^{1 / 2}$ as $u^{p, \varepsilon}(x)$ can be discontinuous across the macro elements $K$ (see [3, Thm 3.11]).

5.3. A priori estimates: modeling error. In this section we analyze the socalled modeling error, i.e., the term $\sup _{K \in \mathcal{T}_{H}}\left\|a^{0}\left(x_{K}\right)-\bar{a}_{K}^{0}\right\|_{F}$ in Theorem 5.11 (see also (5.16) and (5.21)). We recall that under assumption (H2) the homogenized tensor $a^{0}$ can be computed explicitly for $x \in \Omega$ as

$$
a_{i j}^{0}(x)=\int_{Y}\left(a_{i j}(x, y)+\sum_{k=1}^{d} a_{i k}(x, y) \frac{\partial \chi^{j}(x, y)}{\partial x}\right) d y, \quad i, j=1, \ldots, d,
$$

where the functions $\chi^{j}(\cdot, y)$ are the solutions of the cell problem [20]

$$
\int_{Y} a(x, y) \nabla \chi^{j}(x, y) \nabla z d y=-\int_{Y} a(x, y) \mathbf{e}_{j} \nabla z d y, \forall z \in W_{p e r}^{1}(Y),
$$


where $\mathbf{e}_{j}$ is the $j$-th basis vector of $\mathbb{R}^{d}$. In view of $(\mathbf{H 2})$, the "slow variable" $x$ here only depends on the macro elements $K \in \mathcal{T}_{H}$.

We shall now distinguish two cases. First, we consider periodic boundary conditions for the micro problems and second, Dirichlet boundary conditions. Thanks to the framework developed in this paper, we can use for the modeling error of the DG-HMM the results obtained for the continuous FE-HMM (see [32, [3], 9], or 6] for a review).

5.3.1. Periodic boundary conditions. We assume that $S^{1}\left(K_{\delta}, \mathcal{T}_{h}\right) \subset W_{\text {per }}^{1}\left(K_{\delta}\right)$. In this case we suppose that $\delta / \varepsilon \in \mathbb{N}$, i.e., the sampling domain covers an integer number of the exact period of the tensor $a(x, \cdot)$.

Theorem 5.13. Let $u^{0}, u^{H}$ be the solutions of (2.3) and (3.11), respectively, where the micro problems (2.6) are solved using periodic boundary conditions (2.9). Assume $\delta / \varepsilon \in \mathbb{N}$ and that the hypotheses of Theorem 5.11 hold. Then

$$
\begin{array}{r}
\left\|u^{0}-u^{H}\right\| \leq C\left(H+\left(\frac{h}{\varepsilon}\right)^{2}\right), \\
\left\|u^{0}-u^{H}\right\|_{L^{2}(\Omega)} \leq C\left(H^{2}+\left(\frac{h}{\varepsilon}\right)^{2}\right),
\end{array}
$$

where $C$ is independent of $H, h$ and $\varepsilon$.

Proof. Consider, for a given $K \in \mathcal{T}_{H}, \bar{a}_{K}^{0}$ defined in (5.4) with $a^{\varepsilon}(x)=a(x, x / \varepsilon)=$ $a(x, y)$ ( $Y$ periodic in the $y$ variable). As the tensor is constant in the $x$ variable within each element $K \in \mathcal{T}_{H}$, using the change of variable $y=x / \varepsilon$ and the periodicity in the fast variable $y$, we see (compare (5.2) with (5.29) $)$ that $\bar{a}_{K}^{0}(x)$ is equal to the tensor $\left.a^{0}(x)\right|_{K}$ defined in (5.28). This proves the theorem.

Remark 5.14. For a more general tensor $a^{\varepsilon}=a(x, x / \varepsilon)=a(x, y), Y$-periodic in $y$ and not necessarily constant in the slow variable within a macro element $K$, the results of Theorem 5.13 for the modeling error are still valid, provided that we collocate the slow variable of the oscillating tensor at the barycenter $x_{K}$ in the DG-HMM bilinear form, i.e., provided that $a\left(x_{K}, x / \varepsilon\right)$ is used in each sampling domain $K_{\delta}$ instead of $a(x, x / \varepsilon)$ in the bilinear form (3.11). Without collocating the slow variable, we have an additional error term proportional to $\delta$ (see [6]).

5.3.2. Dirichlet boundary conditions. Here we assume that $\delta>\varepsilon$ and $S^{1}\left(K_{\delta}, \mathcal{T}_{h}\right) \subset$ $H_{0}^{1}\left(K_{\delta}\right)$. In this situation $\delta / \varepsilon \in \mathbb{N}$ is no longer required.

Theorem 5.15. Let $u^{0}, u^{H}$ be the solutions of (2.3) and (3.11), respectively, where the micro problems (2.6) are solved using Dirichlet boundary conditions (2.10). Assume that the hypotheses of Theorem 5.11 hold. Then

$$
\begin{array}{r}
\left\|u^{0}-u^{H}\right\| \leq C\left(H+\left(\frac{h}{\varepsilon}\right)^{2}+\frac{\varepsilon}{\delta}\right), \\
\left\|u^{0}-u^{H}\right\|_{L^{2}(\Omega)} \leq C\left(H^{2}+\left(\frac{h}{\varepsilon}\right)^{2}+\frac{\varepsilon}{\delta}\right),
\end{array}
$$

where $C$ is independent of $H, h$ and $\varepsilon$. 
Proof. We have to estimate $\sup _{K \in \mathcal{T}_{H}}\left\|a^{0}\left(x_{K}\right)-\bar{a}_{K}^{0}\right\|_{F}$ where periodic boundary conditions are used for $a^{0}\left(x_{K}\right)$ and Dirichlet boundary conditions are used for $\bar{a}_{K}^{0}$. The bound $\left\|a^{0}\left(x_{K}\right)-\bar{a}_{K}^{0}\right\|_{F} \leq C\left(\frac{\varepsilon}{\delta}\right)$ is deduced from [32, Thm 3.2].

Remark 5.16. For a more general tensor $a^{\varepsilon}=a(x, x / \varepsilon)=a(x, y), Y$-periodic in $y$ and not necessarily constant in the slow variable within a macro element $K$, the results of Theorem 5.13 are still valid with an additional error term proportional to $\delta$, i.e., a modeling error of the form $\delta+\frac{\varepsilon}{\delta}$. This is precisely the modeling error obtained in [32, Thm 3.2].

5.4. Higher order DG-HMM. In this section we explain how to extend the DG-HMM to higher order FEs. We consider

$$
V_{H}^{\ell}\left(\Omega, \mathcal{T}_{H}\right)=\left\{v^{H} \in L^{2}(\Omega) ;\left.v^{H}\right|_{K} \in \mathcal{P}^{\ell}(K), \forall K \in \mathcal{T}_{H}\right\},
$$

where $\mathcal{P}^{\ell}(K)$ is defined as in Section 2.2, Let $\left\{\hat{x}_{j}, \hat{\omega}_{j}\right\}_{j=1}^{J}$ be a QF defined on the reference element $\hat{K}$. While for the analysis we will use hypothesis (H2) (see Section 5.1), we present the algorithm for general tensors. We notice that for simplicial FEs, the assumption (Q2) on the QF (see Section 2.2) implies (Q1). Recall next, assuming (Q2), that the QF $\left\{\hat{x}_{j}, \hat{\omega}_{j}\right\}_{j=1}^{J}$ based on $J$ points is required to be exact for polynomial of degree $2 \ell-2$ if $\ell>1$. It is easy to see that the bounds $J \geq(1 / 2) \ell(\ell+1)(d=2)$ and $J \geq(1 / 6) \ell(\ell+1)(\ell+2)(d=3)$ hold. We notice that some well-known QF minimize the aforementioned inequality in the following sense:

$$
J=\frac{1}{2} \ell(\ell+1), d=2, J=\frac{1}{6} \ell(\ell+1)(\ell+2), d=3 .
$$

For a general discussion on the aforementioned condition we refer to [46] and for examples of QF formula, we refer, e.g., to [34, Chap. 8]. It is clear that the condition (5.35) is desirable as it minimizes the number of quadrature points in the DG-HMM and thus reduces the computational cost (recall that the number of quadrature points is related to the number of micro problems that need to be solved in each macro element). In the analysis below we will assume that (5.35) holds. The algorithm itself described below for higher order macro elements (DG-HMM) can be generalized for QF which do not satisfy (5.35) but the analysis would involve additional technicalities.

Higher order fluxes. For the above QF and every $K \in \mathcal{T}_{H}$, consider the $J$ micro-functions $v_{K_{j}}^{h}$, solutions of (2.6) on the $J$ sampling domains $K_{\delta_{j}}$ centered at $x_{K_{j}}$. We then consider the interpolation polynomial in $\left(\mathcal{P}^{\ell-1}(K)\right)^{d}$ denoted by $\Pi_{a^{\varepsilon} \nabla v_{K}^{h}}(x)$ such that

$$
\Pi_{a^{\varepsilon} \nabla v_{K}^{h}}\left(x_{K_{j}}\right)=\frac{1}{\left|K_{\delta_{j}}\right|} \int_{K_{\delta_{j}}} a^{\varepsilon} \nabla v_{K_{j}}^{h} d x, \quad j=1, \ldots, J .
$$

We note that for $v \in \mathcal{C}^{0}(K)$, assuming (Q2), the following interpolation problem has a unique solution: find $\Pi_{v}(x) \in\left(\mathcal{P}^{\ell-1}(K)\right)^{d}$ s.t. $\Pi_{v}\left(x_{K_{j}}\right)=v\left(x_{K_{j}}\right), j=1, \ldots, J$. Using Corollary 5.5 (for each sampling domain) we see that

$$
\Pi_{a^{\varepsilon} \nabla v_{K}^{h}}\left(x_{K_{j}}\right)=a_{K_{j}}^{0} \nabla v^{H}\left(x_{K_{j}}\right), j=1, \ldots, J,
$$


where $a_{K_{j}}^{0}$ is defined as in (5.3) for the sampling domain $K_{\delta_{j}}$. Similarly to (3.9) we define for each interior interface $e$ of two triangles $K^{+}, K^{-}$an average by

$$
\left\{\Pi_{a^{\varepsilon} \nabla v^{h}}\right\}(s)=\frac{1}{2}\left(\Pi_{a^{\varepsilon} \nabla v_{K^{+}}^{h}}(s)+\Pi_{a^{\varepsilon} \nabla v_{K^{-}}^{h}}(s)\right),
$$

and

$$
\left\{\Pi_{a^{\varepsilon} \nabla v^{h}}\right\}(s)=\Pi_{a^{\varepsilon} \nabla v_{K}^{h}}(s),
$$

where $s \in e$ for a boundary interface $e \in \mathcal{E}_{B}$ of a macro element $K$. When denoting the average $\left\{\Pi_{a^{\varepsilon} \nabla v^{h}}\right\}$ we skip the subscript $K$ for the function $v^{h}$ as the average involves the domain $K^{+} \cup K^{-}$.

We then define the following macro bilinear form on $V_{H}^{\ell}\left(\Omega, \mathcal{T}_{H}\right) \times V_{H}^{\ell}\left(\Omega, \mathcal{T}_{H}\right)$

$$
\begin{aligned}
B_{D G}\left(u^{H}, v^{H}\right) & =\sum_{K \in \mathcal{T}_{H}} \sum_{j=1}^{J} \frac{\omega_{K_{j}}}{\left|K_{\delta_{j}}\right|} \int_{K_{\delta_{j}}} a^{\varepsilon}(x) \nabla u_{K_{j}}^{h} \cdot \nabla v_{K_{j}}^{h} d x-\int_{\Gamma}\left(\left\{\Pi_{a^{\varepsilon} \nabla u^{h}}\right\} \llbracket v^{H} \rrbracket\right. \\
& \left.+\left\{\Pi_{a^{\varepsilon} \nabla v^{h}}\right\} \llbracket u^{H} \rrbracket\right) d s+\int_{\Gamma} \mu \llbracket u^{H} \rrbracket \llbracket v^{H} \rrbracket d s,
\end{aligned}
$$

where $u_{K_{j}}^{h}, v_{K_{j}}^{h}$ are given by the solution of the micro problems (2.6) in the micro FE space $S^{q}\left(K_{\delta_{j}}, \mathcal{T}_{h}\right)$. The penalty weighting function $\mu$ is defined as in (3.10). Notice that the parameter $\alpha$ depends on the polynomial degree of the macro FE space (see 33 for a discussion on this topic). Similarly to (3.11) the macro problem reads: find $u^{H} \in V_{H}^{\ell}\left(\Omega, \mathcal{T}_{H}\right)$ such that

$$
B_{D G}\left(u^{H}, v^{H}\right)=\int_{\Omega} f v^{H} d x, \quad \forall v^{H} \in V_{H}^{\ell}\left(\Omega, \mathcal{T}_{H}\right) .
$$

Remark 5.17. Observe that for piecewise linear polynomials, i.e., $\ell=1$ the definitions (5.38) and (5.39) coincide with (3.9) and (3.10).

Existence and uniqueness. We start with the following generalization of Lemma 4.3 .

Lemma 5.18. Let $v^{H}, w^{H} \in V_{H}^{\ell}\left(\Omega, \mathcal{T}_{H}\right)$ and let $v_{K_{j}}^{h}, j=1, \ldots, J, K \in \mathcal{T}_{H}$ be the solutions of (2.6) constrained by $v_{\text {lin }, K_{j}}^{H}$ with boundary conditions given by (2.9) or (2.10). Assume that (2.2) and (H2) hold and that the QF formula satisfies (Q2). Then

$$
\int_{\Gamma}\left\{\Pi_{a^{\varepsilon} \nabla v^{h}}\right\} \llbracket w^{H} \rrbracket d s \leq C \alpha^{-1 / 2}\left\|\nabla v^{H}\right\|_{L^{2}(\Omega)}\left|w^{H}\right|_{*},
$$

where $\alpha$ is the penalty parameter (see (3.10) ) and where the constant $C$ is independent of $H, h$ and $\varepsilon$.

Proof. By hypothesis (H2), the tensor $a_{K_{j}}^{0}=: a_{K}^{0}$ is constant in each $K \in \mathcal{T}_{H}$, thus, using (5.37) we have $\Pi_{a^{\varepsilon} \nabla v_{K}^{h}}\left(x_{K_{j}}\right)=a_{K}^{0} \nabla v^{H}\left(x_{K_{j}}\right)$. Then in view of (4.6) and (5.37), the proof of Lemma 5.18 is obtained by following the lines of Lemma 4.3 .

Using Lemma 5.18 we obtain the existence and uniqueness of a solution $u^{H} \in$ $V_{H}^{\ell}\left(\Omega, \mathcal{T}_{H}\right)$ of the problem (5.40) by following the lines of Theorem 4.4 , 
A priori error estimates. We define by $\Pi_{a_{K}^{0} \nabla v^{H}}$ the polynomial in $\left(P^{\ell-1}(K)\right)^{d}$ defined by

$$
\Pi_{a_{K}^{0} \nabla v^{H}}\left(x_{K_{j}}\right)=a_{K_{j}}^{0} \nabla v^{H}\left(x_{K_{j}}\right), \quad j=1, \ldots, J
$$

and

$$
\left\{\Pi_{a_{K}^{0} \nabla v^{H}}\right\}(s)=\frac{1}{2}\left(\Pi_{a_{K^{+}}^{0} \nabla v^{H}}(s)+\Pi_{a_{K^{-}}^{0} \nabla v^{H}}(s)\right) .
$$

In view of (5.37) we see that

$$
\Pi_{a^{\varepsilon} \nabla v_{K}^{h}}(s)=\Pi_{a_{K}^{0} \nabla v^{H}}(s) \text { and thus }\left\{\Pi_{a^{\varepsilon} \nabla v^{h}}\right\}=\left\{\Pi_{a_{K}^{0} \nabla v^{H}}\right\} .
$$

Then, similarly to (5.13), the DG-HMM bilinear form (5.39) can be reformulated as

$$
\begin{aligned}
B_{D G}\left(u^{H}, v^{H}\right) & =\sum_{K \in \mathcal{T}_{H}} \sum_{j=1}^{J} \omega_{K_{j}} a_{K_{j}}^{0} \nabla u^{H}\left(x_{K_{j}}\right) \cdot \nabla v^{H}\left(x_{K_{j}}\right) d x \\
& -\int_{\Gamma}\left(\left\{\Pi_{a_{K}^{0} \nabla u^{H}}\right\} \llbracket v^{H} \rrbracket+\left\{\Pi_{a_{K}^{0} \nabla v^{H}}\right\} \llbracket u^{H} \rrbracket\right) d s+\int_{\Gamma} \mu \llbracket u^{H} \rrbracket \llbracket v^{H} \rrbracket d s .
\end{aligned}
$$

We recall the decomposition

$$
\left\|u^{0}-u^{H}\right\| \leq\left\|u^{0}-u^{0, H}\right\| \mid+\left\|u^{0, H}-u^{H}\right\| \|
$$

where $u^{0}$ is the solution of (2.3),$u^{0, H}$ is the solution of (5.15) in $V_{H}^{\ell}\left(\Omega, \mathcal{T}_{H}\right)$ and $u^{H}$ is the solution of (5.40).

As previously, using (H2) (i.e., $a_{K_{j}}^{0}=: a_{K}^{0}$ for all $j=1, \ldots, J$ ), the macro error can be estimated by standard results ([17]) and we obtain

$$
\begin{gathered}
\left\|u^{0}-u^{0, H}\right\| \leq C H^{\ell}, \\
\left\|u^{0}-u^{0, H}\right\|_{L^{2}(\Omega)} \leq C H^{\ell+1},
\end{gathered}
$$

provided that the solution $u^{0}$ of (2.3) is regular enough. For the micro error and the modeling error, we notice that the estimates previously derived only depend on the average of micro problems in sampling domains or analogously on (5.3), (5.4). In view of the reformulation (5.45) of the DG-HMM the results derived for piecewise linear macro FEM can be generalized mutatis mutandis to higher order macro FEM. In particular, following the lines of the proof of Lemma 5.10, assuming the hypotheses of Lemma 5.18, we obtain

$$
\left\|u^{0, H}-u^{H}\right\| \leq C \sup _{K \in \mathcal{T}_{H}}\left\|a^{0}\left(x_{K}\right)-a_{K}^{0}\right\|_{F}\left\|u^{H}\right\| \|
$$

where the constant $C$ is independent of $H, h$ and $\varepsilon$. Considering then the decomposition (5.21), we obtain

$$
\begin{aligned}
& \left\|u^{0}-u^{H}\right\| \leq C\left(H^{\ell}+\left(\frac{h}{\varepsilon}\right)^{2 q}+\sup _{K \in \mathcal{T}_{H}}\left\|a^{0}-\bar{a}_{K}^{0}\right\|_{F}\right), \\
& \left\|u^{0}-u^{H}\right\|_{L^{2}(\Omega)} \leq C\left(H^{\ell+1}+\left(\frac{h}{\varepsilon}\right)^{2 q}+\sup _{K \in \mathcal{T}_{H}}\left\|a^{0}-\bar{a}_{K}^{0}\right\|_{F}\right),
\end{aligned}
$$

which follow from (5.47), (5.48), (5.51), (5.9). We recall that the value of $q$ depends on the regularity of the micro problem (5.1) and the structure of the oscillating tensor $a^{\varepsilon}$. In the above estimate we have also assumed the regularity $u^{0} \in H^{\ell+1}(\Omega)$. For discontinuous coefficients the regularity of the solution may not allow to take 
advantage of high order macro FEs (see Remark [5.9). For smooth solutions if $u^{0} \in H^{k+1}$, then the macroscopic order of convergence is (as usual) proportional to $H^{\min (\ell, k)}\left(\|||||\right.$ norm) and is proportional to $H^{\min (\ell, k)+1}$ ( $L^{2}$ norm). Finally, the last contribution to the error $\left\|a^{0}-\bar{a}_{K}^{0}\right\|_{F}$ in (5.50) and (5.51) can be estimated similarly as in section 5.3 .

\section{Conclusion}

In this paper we have presented a fully discrete analysis of the DG-HMM first introduced in [6]. We have analyzed the method under various coupling conditions between the macro and micro solvers and presented the algorithm for a general oscillating tensor. We have derived optimal $L^{2}$ and $H^{1}$ a priori error estimates. The framework that we introduced for the analysis allows for generalization to random tensor. Partial results for the FE-HMM with random coefficients have been obtained in [32, Thm. 1.3] (for dimensions $d=1$ and $d=3$ ). These results could be used to estimate the modeling error for the DG-HMM with a homogeneous, ergodic random tensor. The macroscopic error can be estimated following the analysis of this paper (for this class of problems, the homogenized tensor is constant). Other regularity assumptions than used in this paper might, however, be necessary to analyze the microscopic error. Recently, fully discrete results have been obtained for the FE-HMM applied to parabolic problems [14]. It would be interesting to extend these results for the DG-HMM. As the mass matrix is block diagonal for the DG method applied to parabolic problems, one could combine the DG-HMM with explicit stabilized methods [8, 2] in time to obtain an explicit coarse-grained algorithm for parabolic homogenization problems. Finally, we note that a generalization of our method to advection-diffusion problems is of high interest. A multiscale method based on the FE-HMM has already been proposed in [4. However, it is well known that in the advection-dominated regime, continuous FEM exhibit poor stability properties due to non-physical oscillations, polluting the numerical solution. Approximations with DG methods have usually much better stability properties and the analysis of DG-FEMs for such single scale problems has attracted much attention in the past few years (see [38] and the references therein). We think that the method developed in this paper is also promising for such problems.

\section{REFERENCES}

[1] J. Aarnes and B.-O. Heimsund, Multiscale discontinuous Galerkin methods for elliptic problems with multiple scales, Multiscale methods in science and engineering, 1-20, Lect. Notes Comput. Sci. Eng., 44, Springer, Berlin, 2005. MR2161705 (2006c:65096)

[2] A. Abdulle, Fourth order Chebyshev methods with recurrence relation, SIAM J. Sci. Comput., 23 (2002), no. 6, 2041-2054. MR1923724 (2003g:65074)

[3] A. Abdulle, On a-priori error analysis of fully discrete heterogeneous multiscale FEM, SIAM Multiscale Model. Simul., 4 (2005), no. 2, 447-459. MR2162863(2006e:65206)

[4] A. Abdulle, Multiscale methods for advection-diffusion problems, Discrete Contin. Dyn. Syst. (2005), suppl., 11-21. MR.2192655 (2006m:76082)

[5] A. Abdulle, Analysis of a heterogeneous multiscale FEM for problems in elasticity, Math. Models Methods Appl. Sci., 16 (2006), no. 2, 615-635. MR2218216 (2007a:65181)

[6] A. Abdulle, The finite element heterogeneous multiscale method: a computational strategy for multiscale PDEs, GAKUTO Int. Ser. Math. Sci. Appl. 31 (2009), 135-184. MR2590959

[7] A. Abdulle, A priori and a posteriori error analysis for numerical homogenization: a unified framework, to appear in Ser. Contemp. Appl. Math., CAM, World Sci. Publishing, Singapore. 
[8] A. Abdulle and A.A. Medovikov, Second order Chebyshev methods based on orthogonal polynomials, Numer. Math., 90 (2001), no. 1, 1-18. MR.1868760 (2002i:65071)

[9] A. Abdulle and C. Schwab, Heterogeneous multiscale FEM for diffusion problem on rough surfaces, SIAM Multiscale Model. Simul., 3 (2005), no. 1, 195-220. MR2123116|(2005i:65173)

[10] A. Abdulle, B. Engquist, Finite element heterogeneous multiscale methods with near optimal computational complexity, SIAM Multiscale Model. Simul. 6 (2007) no. 4, 1059-1084. MR2393025 (2009c:74078)

[11] A. Abdulle, Multiscale method based on discontinuous Galerkin methods for homogenization problems, C. R. Acad. Sci. Paris, Ser. I 346 (2008), no. 1-2, 97-102. MR.2385064

[12] A. Abdulle and A. Nonnenmacher, A short and versatile finite element multiscale code for homogenization problems, Comput. Methods Appl. Mech. Engrg. 198 (2009), no. 37-40, 2839 2859. MR2567848 (2010j:65224)

[13] A. Abdulle, A. Nonnenmacher, A posteriori error analysis of the heterogeneous multiscale method for homogenization problems, C. R. Acad. Sci. Paris, Ser. I. 347 (2009), no. 17-18, 1081-1086. MR 2554581 (2011a:65368)

[14] A. Abdulle and G. Vilmart, Coupling heterogeneous multiscale FEM with Runge-Kutta methods for parabolic homogenization problems: a fully discrete space-time analysis, preprint submitted for publication.

[15] G. Allaire and R. Brizzi, A multiscale finite element method for numerical homogenization, SIAM Multiscale Model. Simul. 4 (2005), no. 3, 790-812. MR2203941 (2006j:35010)

[16] D. Arnold, An interior penalty finite element method with discontinuous elements, SIAM J. Numer. Anal.19 (1982), no. 4, 742-760. MR664882 (83f:65173)

[17] D. Arnold, F Brezzi, B. Cockburn and D. Marini, Unified analysis of discontinuous Galerkin methods for elliptic problems, SIAM J. Numer. Anal. 39 (2001/02), no. 5, 1749-1779. MR.1885715 (2002k:65183)

[18] I. Babŭska and E. Osborn, Generalized finite element methods: Their performance and their relation to mixed methods, SIAM J. Numer. Anal., 20 (1983), 510-536. MR701094 (84h:65076)

[19] I. Babŭska, G. Caloz, and E. Osborn, Special finite element methods for a class of second order elliptic problems with rough coefficients, SIAM J. Numer. Anal., 31 (1994), 945-981. MR.1286212 (95g:65146)

[20] A. Bensoussan, J.-L. Lions and G. Papanicolaou, Asymptotic analysis for periodic structures, North Holland, Amsterdam, 1978. MR503330 (82h:35001)

[21] L. Bers, F. John, and M. Schechter, Partial differential equations, Lectures in Applied Mathematics, Proceedings of the Summer Seminar, Boulder, CO, 1957. MR0163043 (29:346)

[22] A. Bourgeat and A. Piatnitski, Approximations of effective coefficients in stochastic homogenization, Ann. Inst. H. Poincaré Probab. Statist. 40 (2004), no.2, 153-165. MR.2044813 (2005f:35020)

[23] P. Ciarlet, The finite element method for elliptic problems, Classics in Applied Mathematics, 40., SIAM, Philadelphia, PA, 2002. MR.1930132

[24] D. Cioranescu and P. Donato, An introduction to homogenization, Oxford Lecture Series in Mathematics and its Applications, 17, Oxford University Press, New York, 1999. MR 1765047 (2001j:35019)

[25] S. Chen, W. E, and C.-W. Shu, The heterogeneous multiscale method based on the discontinuous Galerkin method for hyperbolic and parabolic problems, SIAM Multiscale Model. Simul. 3 (2005), no. 4, 871-894. MR.2164241 (2006h:65142)

[26] B. Cockburn, G. Karniadakis and C.-W. Shu, The development of discontinuous Galerkin methods, in: Discontinuous Galerkin Methods. Theory, Computation and Applications, edited by B. Cockburn, G. Karniadakis, and C. W. Shu, Lecture Notes in Computational Science and Engineering, Vol. 11 (Springer-Verlag, Berlin, 2000), 3-50. MR.1842161 (2002e:65002)

[27] B. Cockburn and C.W. Shu, The local discontinuous Galerkin method for time-dependent convection-diffusion systems, SIAM J. Numer. Anal. 35 (1998), no. 6, 2440-2463. MR.1655854 (99j:65163)

[28] E. De Giorgi and S. Spagnolo, Sulla convergenza degli integrali dell'energia per operatori ellittici del secondo ordine, Boll. Un. Mat. Ital., 4 (1973), no. 8, 391-411. MR0348255|(50:753)

[29] R. Du and P.B. Ming, Heterogeneous multiscale finite element method with novel numerical integration schemes, Comm. Math. Sci., 8 (2010), no. 4, 863-885. MR2744910 
[30] M. Dryja, On discontinuous Galerkin methods for elliptic problems with discontinuous coefficients, Comput. Methods Appl. Math. 3 (2003), no. 1, 76-85. MR2002258 (2004i:65120)

[31] W. E and B. Engquist, The heterogeneous multiscale methods, Commun. Math. Sci. 1 (2003), no. 1, 87-132. MR.1979846 (2004b:35019)

[32] W. E, P. Ming and P. Zhang, Analysis of the heterogeneous multiscale method for elliptic homogenization problems, J. Amer. Math. Soc. 18 (2005), no. 1, 121-156. MR2114818 (2005k:65246)

[33] Y. Epshteyn and B. Rivière, Estimation of penalty parameters for symmetric interior penalty Galerkin methods, J. Comput. Appl. Math., 206 (2007), no. 2, 843-872. MR2333718 (2008e:65347)

[34] A. Ern and J.-L. Guermond, Theory and practice of finite elements, Applied Mathematical Sciences, 159, Springer-Verlag, New York, 2004. MR2050138 (2005d:65002)

[35] T-Y. Hou, X-H. Wu and Z. Cai, Convergence of a multiscale finite element method for elliptic problems with rapidly oscillating coefficients, Math. Comp. 68 (1999), no. 227, 913943. MR.1642758 (99i:65126)

[36] Y. Efendiev and T. Y. Hou, Multiscale finite element methods. Theory and applications, Surveys and Tutorials in the Applied Mathematical Sciences, 4, Springer, New York, 2009. MR2477579 (2010h:65224)

[37] V. H. Hoang and S. Schwab, High-dimensional finite elements for elliptic problems with multiple scales, SIAM Multiscale Model. Simul., 3 (2005), no. 1, 168-194. MR2123115 (2005m:65267)

[38] P. Houston, C. Schwab, E. Süli, Discontinuous hp-finite element methods for advectiondiffusion-reaction problems, SIAM J. Numer. Anal., 39, (2002), no. 6, 2133-2163. MR.1897953 (2003d:65108)

[39] V.V. Jikov, S.M. Kozlov and O.A. Oleinik, Homogenization of differential operators and integral functionals, Springer-Verlag, Berlin, 1994. MR.1329546 (96h:35003b)

[40] I.G. Kevrekidis, C.W. Gear, J.M. Hyman, P.G. Kevrekidis, O. Runborg and C. Theodoropoulos, Equation-free, coarse-grained multiscale computation: enabling microscopic simulators to perform system-level analysis, Commun. Math. Sci. 1 (2003), no. 4, 715-762. MR2041455 (2005a:65075)

[41] O.A. Ladyzhenskaya, The boundary value problems of mathematical physics, Applied Mathematical Sciences, 49, Springer-Verlag, New York, 1985. MR793735 (87f:35001)

[42] A.M. Matache and C. Schwab, Two-scale FEM for homogenization problems, M2AN Math. Model. Numer. Anal. 36 (2002), no. 4, 537-572. MR.1932304 (2003h:65162)

[43] F. Murat and L. Tartar $H$-convergence, Topics in the mathematical modelling of composite materials, 21-43, Progr. Nonlinear Differential Equations Appl., 31, Birkhäuser Boston, Boston, MA, 1997. MR.1493039

[44] J.T. Oden and K.S. Vemaganti, Estimation of local modeling error and goal-oriented adaptive modeling of heterogeneous materials. I. Error estimates and adaptive algorithms, J. Comput. Phys. 164 (2000), no. 1, 22-47. MR.1786241 (2001e:74079)

[45] G. Sangalli, Capturing small scales in elliptic problems using a Residual-Free Bubbles Finite Element Method, SIAM Multiscale Model. Simul., 1 (2003), no. 3, 485-503. MR2030161 (2004m:65202)

[46] H. J. Schmid, On cubature formulae with a minimal number of knots, Numer. Math. 31 (1978), 281-297. MR514598 (80a:65058)

[47] E.B. Tadmor, M. Ortiz and R. Phillips, Quasicontinuum analysis of defects in solids, Phil. Mag., A73 (1996), 1529-1563.

[48] K. Terada, N. Kikuchi, A class of general algorithms for multi-scale analyses of heterogeneous media, Comput. Methods Appl. Mech. Engrg. 190 (2001), no. 40-41, 5427-5464. MR1843662 (2002d:74061)

[49] M.F. Wheeler, An elliptic collocation-finite element method with interior penalties, SIAM J. Numer. Anal., 15 (1978), 152-161. MR0471383 (57:11117)

Section of Mathematics, Swiss Federal Institute of Technology, 1015 Lausanne, SWITZERLAND

E-mail address: assyr.abdulle@epfl.ch 